\title{
Fixed-order and merged parton-shower predictions for WW and WWj production at the LHC including NLO QCD and EW corrections
}

\author{
Stephan Bräuer, ${ }^{a}$ Ansgar Denner, ${ }^{b}$ Mathieu Pellen, ${ }^{c}$ Marek Schönherr ${ }^{d}$ \\ and Steffen Schumann ${ }^{a}$ \\ ${ }^{a}$ Georg-August Universität Göttingen, Institut für Theoretische Physik, \\ Friedrich-Hund-Platz 1, 37077 Göttingen, Germany \\ ${ }^{b}$ Universität Würzburg, Institut für Theoretische Physik und Astrophysik, \\ Emil-Hilb-Weg 22, 97074 Würzburg, Germany \\ ${ }^{c}$ University of Cambridge, Cavendish Laboratory, \\ 19 JJ Thomson Avenue, Cambridge CB3 OHE, United Kingdom \\ ${ }^{d}$ Institute for Particle Physics Phenomenology, Durham University, \\ Durham DH1 3LE, United Kingdom \\ E-mail: stephan.braeuer@phys.uni-goettingen.de, \\ ansgar.denner@physik.uni-wuerzburg.de, mpellen@hep.phy.cam.ac.uk, \\ marek.schoenherr@durham.ac.uk, \\ steffen.schumann@phys . uni-goettingen.de
}

ABSTRACT: First, we present a combined analysis of $\mathrm{pp} \rightarrow \mu^{+} \nu_{\mu} \mathrm{e}^{-} \bar{\nu}_{\mathrm{e}}$ and $\mathrm{pp} \rightarrow \mu^{+} \nu_{\mu} \mathrm{e}^{-} \bar{\nu}_{\mathrm{e}} \mathrm{j}$ at next-to-leading order, including both QCD and electroweak corrections. Second, we provide all-order predictions for $\mathrm{pp} \rightarrow \mu^{+} \nu_{\mu} \mathrm{e}^{-} \bar{\nu}_{\mathrm{e}}+$ jets using merged parton-shower simulations that also include approximate EW effects. A fully inclusive sample for WW production is compared to the fixed-order computations for exclusive zero- and one-jet selections. The various higher-order effects are studied in detail at the level of cross sections and differential distributions for realistic experimental set-ups. Our study confirms that merged predictions are significantly more stable than the fixed-order ones in particular regarding ratios between the two processes.

KeYwords: NLO Computations, QCD Phenomenology

ARXIV EPRINT: 2005.12128 


\section{Contents}

1 Introduction 1

2 Features of the calculations $\quad 3$

2.1 Born contributions 3

2.2 QCD corrections 4

2.3 EW corrections 4

2.4 Merged predictions with virtual EW approximation 5

2.5 Validation and technical aspects 8

$\begin{array}{lll}2.6 & \text { Set-up } & 9\end{array}$

3 Numerical results $\quad 11$

$\begin{array}{lll}3.1 & \text { Fixed-order results } & 12\end{array}$

$\begin{array}{lll}3.1 .1 & \text { WW production } & 12\end{array}$

$\begin{array}{lll}3.1 .2 & \text { WWj production } & 16\end{array}$

$\begin{array}{ll}\text { 3.1.3 Ratios of WW and WWj } & 19\end{array}$

3.2 Multi-jet merged results 23

3.2.1 Fixed-order vs. merged results at NLO QCD 23

3.2.2 Including EW corrections via the virtual approximation $\quad 27$

3.2.3 Ratios of $\mathrm{WW}$ and $\mathrm{WWj}$

4 Conclusion $\quad 38$

$\begin{array}{ll}\text { A Fragmentation function } & 39\end{array}$

\section{Introduction}

The Large Hadron Collider (LHC) is entering a precision era with the analysis of the full run 2 data set. Many processes will be measured with an unprecedented accuracy and, in that respect, the consideration and evaluation of all possible theoretical effects is mandatory.

Measurements of $\mathrm{W}^{+} \mathrm{W}^{-}$production have been long on-going, leading to very precise results [1]. They are largely motivated by the search for anomalous triple gauge-boson couplings [2] and in turn provide stringent tests of the Standard Model. However, so far, only a single measurement of di-boson production in association with a jet has been published [3]. Such a measurement is complementary to the di-boson ones as it probes similar physics effects in a different kinematics.

On the theoretical side, many higher-order computations have been performed for $\mathrm{W}^{+} \mathrm{W}^{-}$production in order to match the experimental precision. It started many years 
ago with the calculation of next-to-leading order (NLO) QCD corrections for the production of two $\mathrm{W}$ bosons [4-6]. These have been subsequently matched to parton-shower simulations $[7,8]$. Electroweak (EW) corrections have been computed over several years [9-14]. The NNLO QCD corrections have been obtained a few years ago [15, 16]. These have been recently combined with EW corrections [17] and with parton-shower corrections [18]. Resummed computations [19] as well as the gluon-gluon loop-induced contribution [20, 21] are also available. Very recently, a combination of fixed-order predictions with resummed ones has been presented in ref. [22] for vetoed cross sections and transverse observables. Concerning WWj production, far fewer results are available. Owing to the higher multiplicity the NNLO QCD corrections have not been evaluated yet. However, the NLO QCD corrections are known [23, 24], and merged predictions based on the MINLO prescription have been presented [25]. The NLO EW corrections for on-shell W bosons have been computed recently [26].

The present work focuses on the computation and the combination of NLO corrections of QCD and EW type for the processes pp $\rightarrow \mu^{+} \nu_{\mu} \mathrm{e}^{-} \bar{\nu}_{\mathrm{e}}$ and $\mathrm{pp} \rightarrow \mu^{+} \nu_{\mu} \mathrm{e}^{-} \bar{\nu}_{\mathrm{e}} \mathrm{j}$ at the LHC. For the first time, NLO QCD and NLO EW corrections for the off-shell production of both WW and WWj are presented together. All off-shell, non-resonant, and interference contributions are taken into account. Subsequently, all-order predictions based on multi-jet merged parton-shower simulations as implemented in the SHERPA framework are provided. These predictions also include EW effects by combining them with the NLO QCD merged predictions of different jet multiplicity using the virtual approximation of the EW corrections [27] applied to the dominant part of the QCD corrections. The fully inclusive merged sample for WW production can be used in combination with the zeroand one-jet selections. We also compare these sub-samples using two merging prescriptions against fixed-order predictions, hence providing a deeper insight in the merging procedure.

All results presented in this work have been obtained with the fully automated framework SHERPA+RECOLA [28] in realistic experimental set-ups. In particular, vetoes on extra jets are applied for both processes in order to avoid large $K$ factors. Hadronisation and underlying-event effects are not included in the present study but can easily be incorporated thanks to the SHERPA framework. The results are presented in the form of cross sections and differential distributions. Given the similarity of the $\mathrm{WW}$ and $\mathrm{WWj}$ production processes, we provide ratios of cross sections and differential distributions between the two processes. They deliver useful information concerning the correlations between the two channels. We also state for reference the cross sections of the loop-induced contributions $[20,21,24]$, which can be treated completely independently and simply be added to our results.

This article is organised as follows: in section 2, the features of the calculations are explained. In particular, the various contributions included and the methods used are reviewed. Technical details and the set-ups of the calculations are provided. Section 3 is devoted to the numerical results and their discussion. It is divided into two parts: in section 3.1 the fixed-order predictions are displayed, and in section 3.2 results based on multi-jet merging are presented. In each section, various cross sections and a wide range of differential distributions are discussed. Finally, section 4 contains a short summary and concluding remarks. 

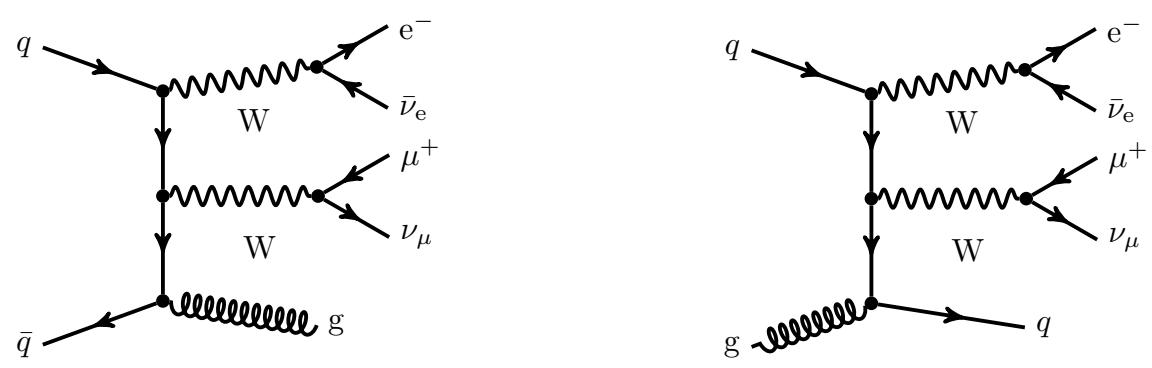

Figure 1. Sample Feynman diagrams for the channels $q \bar{q} \rightarrow \mu^{+} \nu_{\mu} \mathrm{e}^{-} \bar{\nu}_{\mathrm{e}} \mathrm{g}$ (left) and $\mathrm{g} q \rightarrow \mu^{+} \nu_{\mu} \mathrm{e}^{-} \bar{\nu}_{\mathrm{e}} q$ (right), respectively.

\section{Features of the calculations}

In the present computation, we have opted for the 4-flavour scheme. Thus, bottom quarks are treated as massive, and contributions with bottom quarks in the initial state do not appear. Moreover, partonic processes with bottom quarks in the final state are omitted.

\subsection{Born contributions}

In this work, we consider two hadronic processes corresponding to WW and WWj production at the LHC. The first one,

$$
\mathrm{pp} \rightarrow \mu^{+} \nu_{\mu} \mathrm{e}^{-} \bar{\nu}_{\mathrm{e}}
$$

describes the production of two off-shell $\mathrm{W}$ bosons that decay leptonically. The leadingorder (LO) cross section is of order $\mathcal{O}\left(\alpha^{4}\right)$. In the 4-flavour scheme, the contributing partonic channels have initial states $q \bar{q}$ with $q=\mathrm{u}, \mathrm{d}, \mathrm{c}, \mathrm{s}$ and $\gamma \gamma$. However, the photoninduced contribution has not been included in our computations.

The second process involves in addition an extra QCD jet,

$$
\mathrm{pp} \rightarrow \mu^{+} \nu_{\mu} \mathrm{e}^{-} \bar{\nu}_{\mathrm{e}} \mathrm{j}
$$

The dominant partonic channels contribute to the cross section at order $\mathcal{O}\left(\alpha_{\mathrm{s}} \alpha^{4}\right)$, where besides the $q \bar{q}$ channels also contributions from $\mathrm{g} q$ and $\mathrm{g} \bar{q}$ initial states appear. Sample diagrams are shown in figure 1. Subleading contributions of order $\mathcal{O}\left(\alpha^{5}\right)$ originate from initial states $\gamma q$ and $\gamma \bar{q}$, where again $q=\mathrm{u}, \mathrm{d}$, c, s. While always considering the full off-shell production, in the following, both processes are sometimes referred to as $\mathrm{WW}$ and $\mathrm{WWj}$, respectively.

In addition to tree-level contributions, there are also loop-induced contributions with two gluons in the initial state, gg $\rightarrow \mu^{+} \nu_{\mu} \mathrm{e}^{-} \bar{\nu}_{\mathrm{e}}$ and $\mathrm{gg} \rightarrow \mu^{+} \nu_{\mu} \mathrm{e}^{-} \bar{\nu}_{\mathrm{e}}$ g for $\mathrm{WW}$ and $\mathrm{WWj}$, respectively. In section 3 , their LO fiducial cross sections are given for reference but no in-depth analysis is presented. Such contributions are known at NLO for WW [20,21] and have also been studied in detail for WWj [24] where $g q \rightarrow \mu^{+} \nu_{\mu} \mathrm{e}^{-} \bar{\nu}_{\mathrm{e}} q$ contributions have been included. 

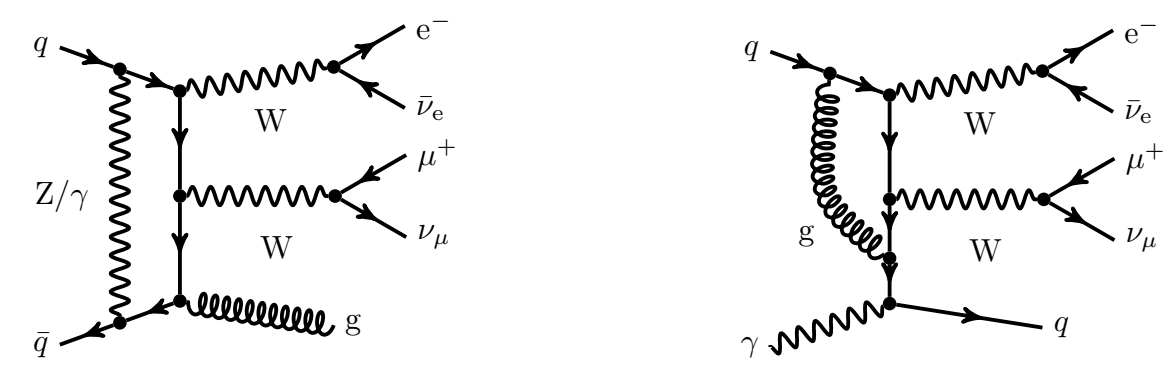

Figure 2. Sample Feynman diagrams representing usual EW corrections of order $\mathcal{O}\left(\alpha_{\mathrm{s}} \alpha^{5}\right)$ in the channel $q \bar{q} \rightarrow \mu^{+} \nu_{\mu} \mathrm{e}^{-} \bar{\nu}_{\mathrm{e}} \mathrm{g}$ (left) and QCD corrections of order $\mathcal{O}\left(\alpha_{\mathrm{s}} \alpha^{5}\right)$ in the channel $\gamma q / \bar{q} \rightarrow$ $\mu^{+} \nu_{\mu} \mathrm{e}^{-} \bar{\nu}_{\mathrm{e}} q / \bar{q}$ (right).

\subsection{QCD corrections}

The QCD corrections to the cross section for WW production are of order $\mathcal{O}\left(\alpha_{\mathrm{s}} \alpha^{4}\right)$, while those for $\mathrm{WWj}$ production are of order $\mathcal{O}\left(\alpha_{\mathrm{s}}^{2} \alpha^{4}\right)$. They consist of real and virtual contributions. The use of the 4-flavour scheme and the exclusion of final states containing bottom quarks avoids contributions such as $\mathrm{gb} \rightarrow \mathrm{W}^{+} \mathrm{W}^{-} \mathrm{b}$ or $\mathrm{gg} \rightarrow \mathrm{W}^{+} \mathrm{W}^{-} \mathrm{b} \overline{\mathrm{b}}$ which are dominated by $\mathrm{Wt}$ and $\mathrm{t} \overline{\mathrm{t}}$ production. Finally, the gg loop-induced contributions are not included in our definition of the NLO QCD predictions.

\subsection{EW corrections}

The EW corrections to WW and WWj production are of order $\mathcal{O}\left(\alpha^{5}\right)$ and $\mathcal{O}\left(\alpha_{\mathrm{s}} \alpha^{5}\right)$, respectively. For both processes, usual EW corrections are included, consisting of virtual corrections as well as real-photon radiation. A sample virtual diagram with the insertion of neutral EW gauge bosons is shown in figure 2 (left).

Given that a recombination algorithm is used to cancel IR divergences differentially, soft and collinear photons are recombined with jets. At NLO EW, these jets are either made of a single gluon or a single quark. This opens the possibility to recombine a soft gluon with a hard photon into a jet which suffers from IR singularities related to soft gluons. These singularities are, by definition, not cancelled by the virtual EW corrections but by virtual QCD corrections to $q \bar{q} \rightarrow \mathrm{W}^{+} \mathrm{W}^{-} \gamma \cdot{ }^{1}$ To deal with such configurations properly, prescriptions for photon-jet separation are needed. For the processes studied here, the related effects are, however, rather suppressed. ${ }^{2}$ Therefore, for the results presented here no prescriptions for photon-jet separation have been used. To justify this procedure, we provide in the appendix a comparison of results obtained without any such prescriptions and a fully consistent approach employing a photon-jet separation based on jet-energy fractions and fragmentation functions following refs. [29-32].

\footnotetext{
${ }^{1}$ Note that in SHERPA the corresponding QCD dipoles are always included by default. These have an underlying Born configuration of the form $a+b \rightarrow \mu^{+} \nu_{\mu} \mathrm{e}^{-} \bar{\nu}_{\mathrm{e}} \gamma$ with $a$ and $b$ QCD partons. However, such configurations would never be accepted by the selector function that requires at least one QCD jet in the final state.

${ }^{2}$ In practice, the IR singularities are regulated by technical cuts, but the dependence on these cuts is very small.
} 


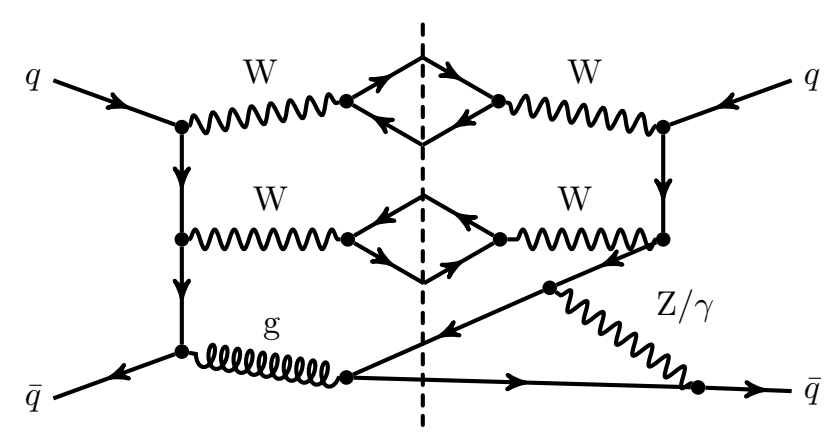

Figure 3. A squared sample diagram representing interference contributions in the real corrections at order $\mathcal{O}\left(\alpha_{\mathrm{s}} \alpha^{5}\right)$ in the channel pp $\rightarrow \mu^{+} \nu_{\mu} \mathrm{e}^{-} \bar{\nu}_{\mathrm{e}} \mathrm{jj}$.

For WWj production, another type of contributions appears in the real corrections, namely interferences between diagrams of orders $\mathcal{O}\left(g_{\mathrm{s}}^{2} g^{4}\right)$ and $\mathcal{O}\left(g^{6}\right)$ for $q \bar{q} \rightarrow \mu^{+} \nu_{\mu} \mathrm{e}^{-} \bar{\nu}_{\mathrm{e}} q^{\prime} \bar{q}^{\prime}$. These contributions are IR-finite and have two (anti-)quarks in both the initial and final state. An example of such an interference term is shown in figure 3.

Also, the QCD corrections to the photon-induced processes in pp $\rightarrow \mu^{+} \nu_{\mu} \mathrm{e}^{-} \bar{\nu}_{\mathrm{ej}}$ are counted as part of the EW corrections. Indeed, even if these are QCD corrections to the underlying process of order $\mathcal{O}\left(\alpha^{5}\right), \gamma q \rightarrow \mu^{+} \nu_{\mu} \mathrm{e}^{-} \bar{\nu}_{\mathrm{e}} q$, they give rise to contributions of order $\mathcal{O}\left(\alpha_{\mathrm{s}} \alpha^{5}\right)$, i.e. of the same order as the EW corrections to the dominant LO contributions. One example of such contributions is shown in figure 2 (right).

Finally, there are further EW corrections for WWj of order $\mathcal{O}\left(\alpha^{6}\right)$. They result from pure EW contributions to partonic processes $q \bar{q} \rightarrow \mu^{+} \nu_{\mu} \mathrm{e}^{-} \bar{\nu}_{\mathrm{e}} q^{\prime} \bar{q}^{\prime}$. They include IR-singular contributions from the splitting $\gamma^{*} \rightarrow q \bar{q}$, which have been first encountered in the computation of QCD corrections to WZ vector-boson scattering [33]. They can be treated using the photon-to-jet conversion function introduced in ref. [34], where a numerical study for $\mathrm{Z}+\mathrm{j}$ production has been presented. This study showed that the corrections of relative order $\mathcal{O}\left(\alpha^{2} / \alpha_{\mathrm{s}}\right)$ are of the order of a per cent for the fiducial cross section and reach up to $10 \%$ for large transverse momenta. Owing to their reduced numerical size, the $\mathcal{O}\left(\alpha^{6}\right)$ corrections have been neglected in the present work.

\subsection{Merged predictions with virtual EW approximation}

Besides calculating $\mathrm{W}$-pair production in association with zero and one jet at fixed-order, we also match both calculations to the parton shower and build a multi-jet merged event simulation that incorporates exact NLO QCD corrections and approximate EW corrections for both the WW and WWj final states, based on the MEPs method [35-38] implemented in the Sherpa Monte Carlo event generator. The aim of the method is to generate an inclusive event sample wherein the hardest $n=0,1, \ldots, n_{\max }$ associated QCD jets are described by $n$-jet matrix elements of the desired LO or NLO accuracy. A measure $Q_{n}$ and a resolution criterion $Q_{\text {cut }}$ are introduced such that $Q_{1}>\ldots>Q_{n}>Q_{\text {cut }}>Q_{n+1}>\ldots$ defines the $n$-jet process and, thus, separates the $n$-jet region from the $n+1$-jet region. The measure $Q_{n}$ is only required to be the clustering scale of an infrared-safe jet algorithm but 
otherwise arbitrary. In practice we choose it to coincide with the parton-shower branching scale $t_{n}$ at the reconstructed splitting.

At LO [35], MePs@Lo, the exclusive cross section with $n<n_{\max }$ jets reads

$$
\mathrm{d} \sigma_{n}^{\mathrm{MEPs} @ \mathrm{Lo}}=\mathrm{d} \Phi_{n} \mathrm{~B}_{n}\left(\Phi_{n}\right) \Theta\left(Q_{n}-Q_{\text {cut }}\right) \mathcal{F}_{n}\left(\mu_{\mathrm{Q}}^{2} ;<Q_{\text {cut }}\right) .
$$

Herein, $\mathrm{B}_{n}$ is the Born matrix element of the $n$-jet process, including all PDF, flux and symmetry/averaging factors, while $\Phi_{n}$ is the $n$-jet phase-space configuration. The $\Theta$-function ensures that all $n$ jets are resolved under the jet criterion $Q_{\text {cut }}$. The parton-shower generating functional $\mathcal{F}_{n}\left(\mu_{\mathrm{Q}}^{2} ;<Q_{\text {cut }}\right)[39]$ applies a truncated vetoed parton shower to the $n$-jet configuration starting at the hard scale $\mu_{\mathrm{Q}}$ and ensures that all further emissions fall into the unresolved region, i.e. $Q_{n+k}<Q_{\text {cut }}(k>0)$. For the highest multiplicity, $n=n_{\max }$, this veto is increased to $Q_{n_{\max }}$ to render the highest multiplicity fully inclusive with respect to additional emissions. The application of this veto also supplies the respective Sudakov form factors to the $n$-jet configuration, resumming the hierarchy of reconstructed partonshower branching scales $t_{1}, \ldots, t_{n}$. In concert with the CKKW scale choice $\mu_{\mathrm{R}}=\mu_{\mathrm{CKKW}}$, defined through [40],

$$
\alpha_{s}^{n}\left(\mu_{\mathrm{CKKW}}^{2}\right)=\alpha_{s}\left(t_{1}\right) \cdots \alpha_{s}\left(t_{n}\right)
$$

and the factorisation and shower-starting scales fixed by the scale of the core process, i.e. $\mu_{\mathrm{F}}=\mu_{\mathrm{Q}}=\mu_{\text {core }}$, a smooth transition across $Q_{\text {cut }}$ is ensured. With these definitions, $\mu_{\text {core }}$ is the only free scale of the CKKW algorithm and fixes the other relevant perturbative scales. In analogy to the fixed-order calculations, the core scale for the reconstructed $\mathrm{pp} \rightarrow \mu^{+} \nu_{\mu} \mathrm{e}^{-} \bar{\nu}_{\mathrm{e}}$ process is chosen as

$$
\mu_{\text {core }}=\frac{1}{2}\left(E_{\mathrm{T}, \mathrm{W}^{+}}+E_{\mathrm{T}, \mathrm{W}^{-}}\right) \equiv \frac{1}{2} \bar{E}_{\mathrm{T}, \mathrm{W}} .
$$

This construction can now be lifted to NLO accuracy in QCD, the MEPs@NLO method [37, 38]. Its exclusive $n$-jet cross section, for $n<n_{\max }^{\mathrm{NLO}}$, based on the MC@NLO expression in refs. [41-43], is defined as

$$
\begin{aligned}
\mathrm{d} \sigma_{n}^{\mathrm{MEPs} @ N \mathrm{NO} Q \mathrm{QCD}}= & \Theta\left(Q_{n}-Q_{\text {cut }}\right)\left[\mathrm{d} \Phi_{n} \overline{\mathrm{B}}_{n}^{\mathrm{QCD}}\left(\Phi_{n}\right) \widetilde{\mathcal{F}}_{n}\left(\mu_{\mathrm{Q}}^{2} ;<Q_{\text {cut }}\right)\right. \\
& \left.+\mathrm{d} \Phi_{n+1} \mathrm{H}_{n}^{\mathrm{QCD}}\left(\Phi_{n+1}\right) \Theta\left(Q_{\text {cut }}-Q_{n+1}\right) \mathcal{F}_{n+1}\left(\mu_{\mathrm{Q}}^{2} ;<Q_{\text {cut }}\right)\right] .
\end{aligned}
$$

Here, $\overline{\mathrm{B}}_{n}^{\mathrm{QCD}}$ describes configurations with $n$ resolved emissions with $Q_{n}>Q_{\text {cut }}$, and takes the form

$$
\overline{\mathrm{B}}_{n}^{\mathrm{QCD}}\left(\Phi_{n}\right)=\mathrm{B}_{n}\left(\Phi_{n}\right)+\tilde{\mathrm{V}}_{n}^{\mathrm{QCD}}\left(\Phi_{n}\right)+\int \mathrm{d} \Phi_{1} \mathrm{D}_{n}^{\mathrm{QCD}}\left(\Phi_{n}, \Phi_{1}\right) \Theta\left(\mu_{\mathrm{Q}}^{2}-t_{n+1}\right) .
$$

It contains QCD NLO renormalised virtual corrections including initial-state mass-factorisation counterterms, $\tilde{\mathrm{V}}_{n}^{\mathrm{QCD}}$, and the integral over the real-emission QCD corrections described by splitting functions in $\mathrm{D}_{n}^{\mathrm{QCD}}$. The functions $\mathrm{D}_{n}^{\mathrm{QCD}}$ are, by construction, also the emission kernels of the fully colour- and spin-correlated parton shower $\widetilde{\mathcal{F}}_{n}[39,41]$ generating the 
$(n+1)$-th emission. The $\mathrm{H}_{n}^{\mathrm{QCD}}$ term corrects its approximate emission pattern to the exact NLO QCD expression. It takes the form

$$
\mathrm{H}_{n}^{\mathrm{QCD}}\left(\Phi_{n+1}\right)=\mathrm{R}_{n}^{\mathrm{QCD}}\left(\Phi_{n+1}\right)-\mathrm{D}_{n}^{\mathrm{QCD}}\left(\Phi_{n}, \Phi_{1}\right) \Theta\left(\mu_{\mathrm{Q}}^{2}-t_{n+1}\right),
$$

with the NLO QCD real-emission matrix element $\mathrm{R}_{n}^{\mathrm{QCD}}$. The quantity $\mathrm{H}_{n}$ is thus a realsubtracted contribution.

When $n_{\max }>n_{\max }^{\mathrm{NLO}}$, i.e. when only the first $n_{\max }^{\mathrm{NLO}}$ emissions can be described at NLO QCD accuracy, the additional $n_{\max }-n_{\max }^{\mathrm{NLO}}$ emissions are added at LO accuracy. In this case the MeNloPs method [36, 38, 39] is used for $n=n_{\max }^{\mathrm{NLO}}+k(k>0)$,

$$
\mathrm{d} \sigma_{n}^{\mathrm{MENLOPS}}=\mathrm{d} \Phi_{n} k_{n_{\max }^{\mathrm{NLO}}}\left(\Phi_{n_{\max }^{\mathrm{NLO}}}\left(\Phi_{n}\right)\right) \mathrm{B}_{n}\left(\Phi_{n}\right) \Theta\left(Q_{n}-Q_{\text {cut }}\right) \mathcal{F}_{n}\left(\mu_{\mathrm{Q}}^{2} ;<Q_{\text {cut }}\right) .
$$

It thus furnishes a local $K$ factor, defined on the highest multiplicity phase space for which NLO corrections are available, $\Phi_{n_{\max }^{\text {NLO }}}$,

$$
k_{n}\left(\Phi_{n}\right)=\frac{\overline{\mathrm{B}}_{n}\left(\Phi_{n}\right)}{\mathrm{B}_{n}\left(\Phi_{n}\right)}\left(1-\frac{\mathrm{H}_{n}\left(\Phi_{n+1}\right)}{\mathrm{B}_{n+1}\left(\Phi_{n+1}\right)}\right)+\frac{\mathrm{H}_{n}\left(\Phi_{n+1}\right)}{\mathrm{B}_{n+1}\left(\Phi_{n+1}\right)},
$$

to the MePs@Lo expression of that multiplicity. The $K$ factor expands to $1+\mathcal{O}\left(\alpha_{\mathrm{s}}\right)$ and, thus, retains both the NLO accuracy of the $n_{\max }^{\mathrm{NLO}}$-parton process and the LO accuracy of the $n$-parton process, while simultaneously guaranteeing a smooth transition across the merging parameter $Q_{\text {cut }}$ for all multiplicities. ${ }^{3}$

Approximate NLO EW corrections are incorporated by replacing the usual NLO QCD $\overline{\mathrm{B}}_{n}$ function of eq. (2.6) with $[27,45]$

$$
\overline{\mathrm{B}}_{n}^{\mathrm{QCD}+\mathrm{EW}}\left(\Phi_{n}\right)=\overline{\mathrm{B}}_{n}^{\mathrm{QCD}}\left(\Phi_{n}\right)+\mathrm{B}_{n}\left(\Phi_{n}\right) \delta_{\mathrm{EW}}^{\mathrm{approx}}\left(\Phi_{n}\right)+\mathrm{B}_{n}^{\mathrm{sub}}\left(\Phi_{n}\right)
$$

in an additive combination of QCD and EW corrections or

$$
\overline{\mathrm{B}}_{n}^{\mathrm{QCD} \times \mathrm{EW}}\left(\Phi_{n}\right)=\overline{\mathrm{B}}_{n}^{\mathrm{QCD}}\left(\Phi_{n}\right)\left(1+\delta_{\mathrm{EW}}^{\mathrm{approx}}\left(\Phi_{n}\right)\right)+\mathrm{B}_{n}^{\mathrm{sub}}\left(\Phi_{n}\right)
$$

in a multiplicative manner. In both cases, the approximate EW correction is defined as

$$
\delta_{\mathrm{EW}}^{\mathrm{approx}}\left(\Phi_{n}\right)=\frac{\mathrm{V}_{n}^{\mathrm{EW}}\left(\Phi_{n}\right)+\mathrm{I}_{n}^{\mathrm{EW}}\left(\Phi_{n}\right)}{\mathrm{B}_{n}\left(\Phi_{n}\right)} .
$$

Herein, $\mathrm{V}_{n}^{\mathrm{EW}}$ represents the renormalised virtual $\mathrm{EW}$ corrections and $\mathrm{I}_{n}^{\mathrm{EW}}$ the approximate EW real-emission corrections integrated over the real-emission phase space. ${ }^{4}$ Finally, $\mathrm{B}_{n}^{\mathrm{sub}}$ are possible Born contributions at subleading orders, which are, however, zero in the processes under consideration in this article. We stress that the modified $\overline{\mathrm{B}}_{n}$ functions and,

\footnotetext{
${ }^{3}$ Reference [44] explored the possibility to substitute the local $K$ factor $k_{n}$ defined on the highest NLO multiplicity by the $K$ factor defined on the lowest NLO multiplicity, i.e. the core process. For the considered processes it found a negligible dependence on this choice for most observables. In the context of employing the virtual EW approximation, however, the higher multiplicity LO processes would then not directly inherit approximate EW corrections for kinematic quantities like the leading jet.

${ }^{4}$ In practice, we use the Catani-Seymour I operator [46] with $\alpha=1$.
} 
thus, the EW corrections also enter the local $K$ factor in eq. (2.10) applied to the highermultiplicity processes. They thus receive approximate EW corrections through $k_{n}$ from the $n_{\text {max }}^{\mathrm{NLO}}$-jet process, guaranteeing their continuity across the merging parameter $Q_{\text {cut }}$ and consistency with respect to whether an additional jet at LO accuracy is merged or not.

It should be noted, however, that both the phase-space-point-wise definition of the additive and multiplicative combinations of eq. (2.11) and (2.12) differ from the usual definition constructed on histogram level in the case of fixed-order calculations. Both constructions apply the approximate EW corrections only to the QCD $\overline{\mathrm{B}}$-function (collecting the virtual corrections and the soft-collinear limit of the real-emission corrections, but not to its hard wide-angle radiation part). Both $\overline{\mathrm{B}}_{n}^{\mathrm{QCD}+\mathrm{EW}}$ and $\overline{\mathrm{B}}_{n}^{\mathrm{QCD} \times \mathrm{EW}}$ are then dressed with QCD radiation through $\widetilde{\mathcal{F}}$. Thus, the results will differ from the bin-by-bin additive and multiplicative combination of QCD and EW corrections of the fixed-order calculation, respectively. Major differences will occur between the two additive combinations if selection criteria acting on additional jet activity (like jet vetoes) are present, while the multiplicative combination will differ substantially if hard real radiation described through the $\mathrm{H}_{n}$ events forms a large part of the event sample. We have checked, however, that the latter is not the case for any observable presented in this paper. In fact, in our set-up for $\mathrm{WW}+$ jets production, the $\mathrm{H}_{n}$-terms' contribution never exceeds $20 \%$ (15\%) for the zerojet (one-jet) selection for inclusive observables and 5\% (5\%) in the EW Sudakov regime. We would like to emphasise that the approximate inclusion of EW corrections does not improve predictions for inclusive observables. It has an intrinsic uncertainty of a few per cent and primarily catches leading logarithmic EW corrections relevant in the high-energy limit.

Finally, the approximation of integrated-out real-emission corrections can be problematic for leptonic observables in particular. Here, radiative energy loss through photon bremsstrahlung can amount to $\mathcal{O}(1)$ effects below the on-shell W-pair production threshold or the $\mathrm{Z}$ pole (see for instance ref. [13]). If not via explicit real-radiation matrix elements, these effects can be included through either QED parton showers or a soft-photon resummation, the latter of which will be employed in this work. It is important to note, however, that both solutions lead to a double counting of virtual QED corrections which remains unresolved. As these corrections are applied to the respective $\mathrm{W}$ decays only, it is ensured that they do not interfere with the EW logarithms in the Sudakov regime [14, 27]. The unitarity of these resummations also ensures that inclusive cross sections remain unaffected.

\subsection{Validation and technical aspects}

The results presented here have been obtained with the combination SHERPA+RECOLA [28] which has already been used for several NLO QCD and EW computations [47-49]. SherPa $[50,51]$ is a multi-purpose Monte Carlo event generator capable to compute both QCD and EW corrections in a general and automated way. It implements the tree-level matrix-element generators Comix [52] and AMEGIC [53] and employs an implementation of the Catani-Seymour dipole subtraction method for both QCD and QED soft and collinear singularities $[46,54]$. To simulate QCD parton cascades it employs the dipole-shower algorithm presented in ref. [55]. For the merging of parton-shower-evolved hard processes 
at tree and one-loop level a truncated-shower approach is employed [35-37]. Higher-order QED corrections are effected through the soft-photon resummation of Yennie, Frautschi, and Suura (YFS) [56], as implemented in ref. [57].

RECOLA $[58,59]$ is a matrix-element generator that provides any one-loop amplitude in the Standard Model. It relies on the CoLLIER library $[60,61]$ to numerically evaluate the one-loop scalar [62-65] and tensor integrals [66-68]. The interface between SHERPA and RECOLA is fully general and hence enables the computation of any NLO cross section in the Standard Model. The interface is compatible with REcola2 [69] which features a considerable reduction of the memory needs when computing many channels at a time. This is made possible by the use of crossing symmetries in order to compute the minimum number of processes and has already been exploited in ref. [70].

The computation of NLO QCD corrections with SHERPA has by now become a standard. However, the possibility to compute one-loop EW corrections in an automated manner is still rather recent [46]. To that end, we have carefully tested the implementation of the EW corrections against an independent program, namely the combination MOCANLO+RECOLA, that has already been used for a variety of processes including $\mathrm{V}+$ jets production $[33,71,72]$.

The SHERPA framework has already been utilised for NLO QCD and EW computations for multi-jet [48], $\mathrm{V}+$ jet $[27,73]$, di-boson [14, 74], tri-boson [47, 75], and $t \bar{t}+$ jet production [45]. Di-boson-production processes, in particular, have been cross-validated among various programs including SHERPA+RECOLA and MoCANLO+RECOLA in ref. [76].

\subsection{Set-up}

Numerical inputs. The predictions presented here are obtained for the LHC operating at $\sqrt{s}=13 \mathrm{TeV}$. For the parton distribution function (PDF), the NNPDF31_nlo_as_0118 _luxqed set [77] is used and interfaced through LHAPDF [78]. It is based on ref. [79] for the extraction of the photon content. The choice of the renormalisation and factorisation scales follows the one of ref. [24] and reads

$$
\mu_{\mathrm{R}}=\mu_{\mathrm{F}}=\frac{1}{2}\left(E_{\mathrm{T}, \mathrm{W}^{+}}+E_{\mathrm{T}, \mathrm{W}^{-}}\right) \equiv \frac{1}{2} \bar{E}_{\mathrm{T}, \mathrm{W}},
$$

with $E_{\mathrm{T}, \mathrm{W}}=\sqrt{M_{\mathrm{W}}^{2}+\left(\vec{p}_{\mathrm{T}, \ell}+\vec{p}_{\mathrm{T}, \nu}\right)^{2}}$. The value of the strong coupling is chosen consistently with the used PDF set, i.e.

$$
\alpha_{\mathrm{S}}\left(M_{\mathrm{Z}}^{2}\right)=0.118
$$

To fix the EW coupling, the $G_{\mu}$ scheme $[80,81]$ is employed throughout with

$$
\alpha=\frac{\sqrt{2}}{\pi} G_{\mu} M_{\mathrm{W}}^{2}\left(1-\frac{M_{\mathrm{W}}^{2}}{M_{\mathrm{Z}}^{2}}\right) \quad \text { and } \quad G_{\mu}=1.16637 \times 10^{-5} \mathrm{GeV}^{-2}
$$


We use the following values for the masses and widths:

$$
\begin{aligned}
m_{\mathrm{t}} & =173.21 \mathrm{GeV}, & m_{\mathrm{b}} & =4.8 \mathrm{GeV}, \\
M_{\mathrm{Z}}^{\mathrm{OS}} & =91.1876 \mathrm{GeV}, & \Gamma_{\mathrm{Z}}^{\mathrm{OS}} & =2.4952 \mathrm{GeV}, \\
M_{\mathrm{W}}^{\mathrm{OS}} & =80.385 \mathrm{GeV}, & \Gamma_{\mathrm{W}}^{\mathrm{OS}} & =2.085 \mathrm{GeV}, \\
M_{\mathrm{H}} & =125.0 \mathrm{GeV}, & \Gamma_{\mathrm{H}} & =4.07 \times 10^{-3} \mathrm{GeV} .
\end{aligned}
$$

Both the top- and bottom-quark widths are taken to be zero as these particles do not appear as resonances in our computations. The values for the Higgs-boson mass and width follow the recommendations of the Higgs cross section working group [82]. The pole masses and widths used for the simulations are obtained from the measured on-shell (OS) values for the $\mathrm{W}$ and $\mathrm{Z}$ bosons according to [83]

$$
M_{\mathrm{V}}=\frac{M_{\mathrm{V}}^{\mathrm{OS}}}{\sqrt{1+\left(\Gamma_{\mathrm{V}}^{\mathrm{OS}} / M_{\mathrm{V}}^{\mathrm{OS}}\right)^{2}}}, \quad \Gamma_{\mathrm{V}}=\frac{\Gamma_{\mathrm{V}}^{\mathrm{OS}}}{\sqrt{1+\left(\Gamma_{\mathrm{V}}^{\mathrm{OS}} / M_{\mathrm{V}}^{\mathrm{OS}}\right)^{2}}},
$$

with $\mathrm{V}=\mathrm{W}, \mathrm{Z}$.

Event selection. The event-selection criteria are based on ref. [3] and are specified for both processes in the following. To cluster QCD jets we use the anti- $k_{\mathrm{T}}$ algorithm [84] with a jet-resolution parameter of $R=0.4$. Photons are recombined with charged leptons and QCD jets using a standard cone algorithm with a radius parameter of $R=0.1$.

- $\mathrm{pp} \rightarrow \mu^{+} \nu_{\mu} \mathrm{e}^{-} \bar{\nu}_{\mathrm{e}}$

The charged leptons are required to fulfil

$$
p_{\mathrm{T}, \ell}>20 \mathrm{GeV}, \quad\left|y_{\ell}\right|<2.5, \quad \Delta R_{\ell \ell^{\prime}}>0.1,
$$

as well as

$$
m_{\ell \ell}>10 \mathrm{GeV}, \quad p_{\mathrm{T}, \mathrm{miss}}>20 \mathrm{GeV} .
$$

In addition, a jet-veto is applied in order to limit the size of the QCD corrections. In particular, any event with an identified jet such that

$$
p_{\mathrm{T}, \mathrm{j}}>25 \mathrm{GeV}=p_{\mathrm{T}, \mathrm{j}, \mathrm{cut}} \quad \text { and } \quad\left|y_{\mathrm{j}}\right|<2.5
$$

is rejected.

- $\mathrm{pp} \rightarrow \mu^{+} \nu_{\mu} \mathrm{e}^{-} \bar{\nu}_{\mathrm{e}} \mathrm{j}$

For the production of two off-shell $\mathrm{W}$ bosons in association with a jet, the lepton cuts (2.19) and (2.20) are preserved. In addition, one jet has to fulfil eq. (2.21) and

$$
\Delta R_{\ell^{ \pm} \mathrm{j}}>0.4 .
$$

A veto with the same parameters is then applied on the occurrence of any additional jet. 
The implementation of the applied jet veto follows the experimental analysis in ref. [3] and is not driven by theoretical considerations. Note that experimentally, a further veto on b-jets is usually applied, both, for WW and WWj production, thereby eliminating contributions from single-top and top-pair production. However, as we exclude events with final-state bottom quarks in our computation, i.e. resonant top-quark propagators, there is no need to apply such a veto here. The listed event selections and the observable calculations for the results presented in the following have been implemented in RIVET [85].

MePs@Nlo calculation. For the MePs@NLO predictions we merge the NLO QCD matrix elements for pp $\rightarrow \mu^{+} \nu_{\mu} \mathrm{e}^{-} \bar{\nu}_{\mathrm{e}}$ and $\mathrm{pp} \rightarrow \mu^{+} \nu_{\mu} \mathrm{e}^{-} \bar{\nu}_{\mathrm{e}} \mathrm{j}$ and the tree-level matrix elements for $\mathrm{pp} \rightarrow \mu^{+} \nu_{\mu} \mathrm{e}^{-} \bar{\nu}_{\mathrm{e}} \mathrm{jj}$ and $\mathrm{pp} \rightarrow \mu^{+} \nu_{\mu} \mathrm{e}^{-} \bar{\nu}_{\mathrm{e}} \mathrm{jjj}$, provided by Comix [52]. The merging scale is set to

$$
Q_{\text {cut }}=30 \mathrm{GeV} \text {. }
$$

Per default we use the CKKW scale-setting prescription of eqs. (2.4) and (2.5) to define the renormalisation, factorisation, and resummation scales, with the scale of the inner core process, $\mu_{\text {core }}$, given by

$$
\mu_{\text {core }}=\frac{1}{2} \bar{E}_{\mathrm{T}, \mathrm{W}} .
$$

As discussed in section 2.4, this directly fixes the renormalisation scale to the CKKW scale, $\mu_{\mathrm{R}}=\mu_{\mathrm{CKKW}}$, and the factorisation and parton-shower starting scale to the core scale $\mu_{\mathrm{F}}=\mu_{\mathrm{Q}}=\mu_{\text {core }}$. We describe the $\mathrm{WW}$ and $\mathrm{WWj}$ production processes at NLO QCD, i.e. $n_{\max }^{\mathrm{NLO}}=1$ and evaluate approximate NLO EW corrections up to this order. LO contributions are taken into account for WWjj and WWjjj production processes, i.e. $n_{\max }=3$, that are subject to local $K$ factors, cf. eq. (2.10). All Standard Model parameters and event-selection criteria are defined as detailed above, thus, in compliance with the fixed-order calculations.

\section{Numerical results}

In this section, we discuss the numerical results obtained for the processes $\mathrm{pp} \rightarrow \mu^{+} \nu_{\mu} \mathrm{e}^{-} \bar{\nu}_{\mathrm{e}}$ and $\mathrm{pp} \rightarrow \mu^{+} \nu_{\mu} \mathrm{e}^{-} \bar{\nu}_{\mathrm{e}} \mathrm{j}$. We present both, fiducial cross sections and differential distributions. We provide theoretical predictions at LO, NLO QCD and EW, as well as MEPs@NLO incorporating EW corrections in the virtual EW approximation. Particular emphasis is put on the combination of QCD and EW corrections and the impact of the QCD parton shower.

In this article, the NLO QCD and EW cross sections are defined as

$$
\sigma_{\mathrm{QCD}}^{\mathrm{NLO}}=\sigma^{\mathrm{Born}}\left(1+\delta_{\mathrm{QCD}}^{\mathrm{NLO}}\right) \quad \text { and } \quad \sigma_{\mathrm{EW}}^{\mathrm{NLO}}=\sigma^{\mathrm{Born}}\left(1+\delta_{\mathrm{EW}}^{\mathrm{NLO}}\right),
$$

respectively. The additive prescription to combine QCD and EW corrections reads

$$
\sigma_{\mathrm{QCD}+\mathrm{EW}}^{\mathrm{NLO}}=\sigma^{\mathrm{Born}}\left(1+\delta_{\mathrm{QCD}}^{\mathrm{NLO}}+\delta_{\mathrm{EW}}^{\mathrm{NLO}}\right)
$$


while the multiplicative one is defined as

$$
\begin{aligned}
\sigma_{\mathrm{QCD} \times \mathrm{EW}}^{\mathrm{NLO}} & =\sigma^{\mathrm{Born}}\left(1+\delta_{\mathrm{QCD}}^{\mathrm{NLO}}\right)\left(1+\delta_{\mathrm{EW}}^{\mathrm{NLO}}\right) \\
& =\sigma_{\mathrm{QCD}}^{\mathrm{NLO}}\left(1+\delta_{\mathrm{EW}}^{\mathrm{NLO}}\right)=\sigma_{\mathrm{EW}}^{\mathrm{NLO}}\left(1+\delta_{\mathrm{QCD}}^{\mathrm{NLO}}\right) .
\end{aligned}
$$

The difference between these two prescriptions could be used as an estimate of the missing QCD-EW mixed corrections. In this context, the NLO QCD $\times$ EW combination can be understood as an improved prediction when the typical scales of the QCD and EW corrections are well separated. In the following, we argue that this is the case for the processes at hand.

For the first time, we also present predictions based on a multiplicative scheme, cf. eq. (2.12), to implement approximate NLO EW corrections in merged calculations of NLO QCD matrix elements for WW and WWj production matched to the SHERPA parton shower. In addition, to validate the virtual EW approximation employed in the multijetmerged calculations, we study

$$
\sigma_{\mathrm{QCD} \times \mathrm{EW}_{\text {approx }}}^{\mathrm{NLO}}=\sigma^{\mathrm{Born}}\left(1+\delta_{\mathrm{QCD}}^{\mathrm{NLO}}\right)\left(1+\delta_{\mathrm{EW}}^{\mathrm{approx}}\right)
$$

with $\delta_{\mathrm{EW}}^{\text {approx }}$ defined in eq. (2.13).

To estimate the theoretical uncertainty of our predictions we consider the usual set of 7-point scale variations, i.e. $\left\{\left(\frac{1}{2} \mu_{\mathrm{R}}, \frac{1}{2} \mu_{\mathrm{F}}\right),\left(\frac{1}{2} \mu_{\mathrm{R}}, \mu_{\mathrm{F}}\right),\left(\mu_{\mathrm{R}}, \frac{1}{2} \mu_{\mathrm{F}}\right),\left(\mu_{\mathrm{R}}, \mu_{\mathrm{F}}\right),\left(\mu_{\mathrm{R}}, 2 \mu_{\mathrm{F}}\right)\right.$, $\left.\left(2 \mu_{\mathrm{R}}, \mu_{\mathrm{F}}\right),\left(2 \mu_{\mathrm{R}}, 2 \mu_{\mathrm{F}}\right)\right\}$. The uncertainties quoted for fiducial cross sections and differential distributions in the following correspond to the resulting envelope. All systematic variations are evaluated on-the-fly using the implementation of the algorithm presented in ref. [86] in the SHERPA framework.

\subsection{Fixed-order results}

\subsubsection{WW production}

In table 1, fiducial cross sections for pp $\rightarrow \mu^{+} \nu_{\mu} \mathrm{e}^{-} \bar{\nu}_{\mathrm{e}}$ at LO, NLO QCD, and NLO EW accuracy are compiled. Thanks to the jet veto, the QCD corrections amount to $+0.4 \%$ only, while the EW corrections reach $-3 \%$. The two prescriptions of combining the QCD and EW corrections lead to practically identical results. The contribution from $\gamma \gamma$ initial states contained in the LO cross section amounts to $4.006(5) \mathrm{fb}$, i.e. $1.3 \%$. The QCD scale uncertainty of the LO prediction is estimated, in the absence of a renormalisation scale dependence, by variations of the factorisation scale by factors of $\frac{1}{2}$ and 2 . For the NLO QCD result we consider the full 7-point variations. For the LO prediction this yields an estimated uncertainty of order $6 \%$, while at NLO QCD it is reduced to the $2 \%$ level for our set-up. However, this reduction depends strongly on the precise form of the jet veto.

In addition to the channels considered in table 1, there exists a loop-induced contribution from the partonic process gg $\rightarrow \mu^{+} \nu_{\mu} \mathrm{e}^{-} \bar{\nu}_{\mathrm{e}}$ at order $\mathcal{O}\left(\alpha_{\mathrm{s}}^{2} \alpha^{4}\right)$. In the present set-up, it amounts to $29.38(1)_{-17.9 \%}^{+24.6 \%}$ fb, i.e. $9.2 \%$ of the LO prediction for $q \bar{q} \rightarrow \mu^{+} \nu_{\mu} \mathrm{e}^{-} \bar{\nu}_{\mathrm{e}}$. 


\begin{tabular}{|c|c|c|c|c|}
\hline$\sigma^{\mathrm{LO}}[\mathrm{fb}]$ & $\sigma_{\mathrm{QCD}}^{\mathrm{NLO}}[\mathrm{fb}]$ & $\sigma_{\mathrm{EW}}^{\mathrm{NLO}}[\mathrm{fb}]$ & $\sigma_{\mathrm{QCD}+\mathrm{EW}}^{\mathrm{NLO}}[\mathrm{fb}]$ & $\sigma_{\mathrm{QCD} \times \mathrm{EW}}^{\mathrm{NLO}}[\mathrm{fb}]$ \\
\hline $319.7(1)_{-6.3 \%}^{+5.2 \%}$ & $321.1(8)_{-2.2 \%}^{+2.1 \%}$ & $310.8(5)$ & $312.2(9)$ & $312.1(9)$ \\
\hline
\end{tabular}

Table 1. Fiducial cross sections for $\mathrm{pp} \rightarrow \mu^{+} \nu_{\mu} \mathrm{e}^{-} \bar{\nu}_{\mathrm{e}}$ at $\sqrt{s}=13 \mathrm{TeV}$ at LO, NLO QCD, and NLO EW. For the combination of NLO QCD and NLO EW corrections results for the additive and multiplicative prescription are quoted.

In the following, several differential distributions are presented. In the upper panels of the plots, absolute predictions at LO, NLO QCD, NLO QCD + EW, and NLO QCD $\times$ $\mathrm{EW}$ accuracy are shown. In addition, $\mathrm{NLO} \mathrm{QCD} \times \mathrm{EW}_{\text {approx }}$ results are displayed to gauge the quality of the approximation to be used when constructing the merged results in section 3.2.2. The lower panels contain the corresponding results normalised to the NLO QCD ones. Accordingly, in what follows we quote corrections/deviations relative to the NLO QCD prediction, corresponding to the ratio plots provided. The scale uncertainty of the NLO QCD prediction, given by the envelope of the 7-point variations of $\mu_{\mathrm{R}}$ and $\mu_{\mathrm{F}}$, is indicated by the green band.

In figure 4, various transverse-momentum observables as well as the distribution in the rapidity of the anti-muon are shown. For the distribution in the transverse momentum of the anti-muon, the effect of QCD corrections is rather large. They tend to lower the predictions for larger transverse momentum and exceed $-40 \%$ at $p_{\mathrm{T}, \mu^{+}}=400 \mathrm{GeV}$. The large negative QCD corrections result from the jet-veto cuts. Owing to the Sudakov logarithms in the virtual corrections, the EW corrections follow the same trend and exceed $-25 \%$ at $400 \mathrm{GeV}$ with respect to the NLO QCD prediction. For the rapidity distribution of the anti-muon, the QCD corrections are moderate throughout, being about $-1 \%$ in the central region, while becoming positive in the peripheral region at a level of $+5 \%$. On the other hand, the EW corrections exceed the estimated NLO QCD scale uncertainty but do not feature a sizeable shape distortion. The distributions in the transverse momentum of the anti-muon-electron system and the missing transverse energy display very similar behaviour both qualitatively and quantitatively. This is explained by the fact that the missing momentum is defined as the sum of the two neutrino momenta. This observable has thus a very similar kinematics as the transverse momentum of the two charged leptons. In both cases, the NLO QCD corrections reach about $-40 \%$ at $400 \mathrm{GeV}$, while the EW ones are of order $-15 \%$ for the same transverse momentum. Around $100 \mathrm{GeV}$ the NLO QCD prediction suddenly exceeds the LO one at a level of $20 \%$. The corrections then turn negative towards high transverse momentum. This can be understood as follows. At LO, contributions with two resonant $\mathrm{W}$ bosons require these bosons to be back-to-back and therefore cannot contribute to events with transverse momenta $p_{\mathrm{T}, \mu^{+} \mathrm{e}^{-}}$or $p_{\mathrm{T} \text {,miss }}$ larger than about $M_{\mathrm{W}}[13,14]$. Thus, at LO such events can only result from contributions with at most one resonant $\mathrm{W}$ boson and are therefore suppressed. At NLO, the momentum of the extra jet can balance the momenta of the two resonant $\mathrm{W}$ bosons allowing for large $p_{\mathrm{T}, \mu^{+} \mathrm{e}^{-}}$and/or $p_{\mathrm{T}, \mathrm{miss}}$ also in the presence of two resonant $\mathrm{W}$ bosons. Going towards higher transverse momenta, such configurations are then suppressed by the jet veto that 

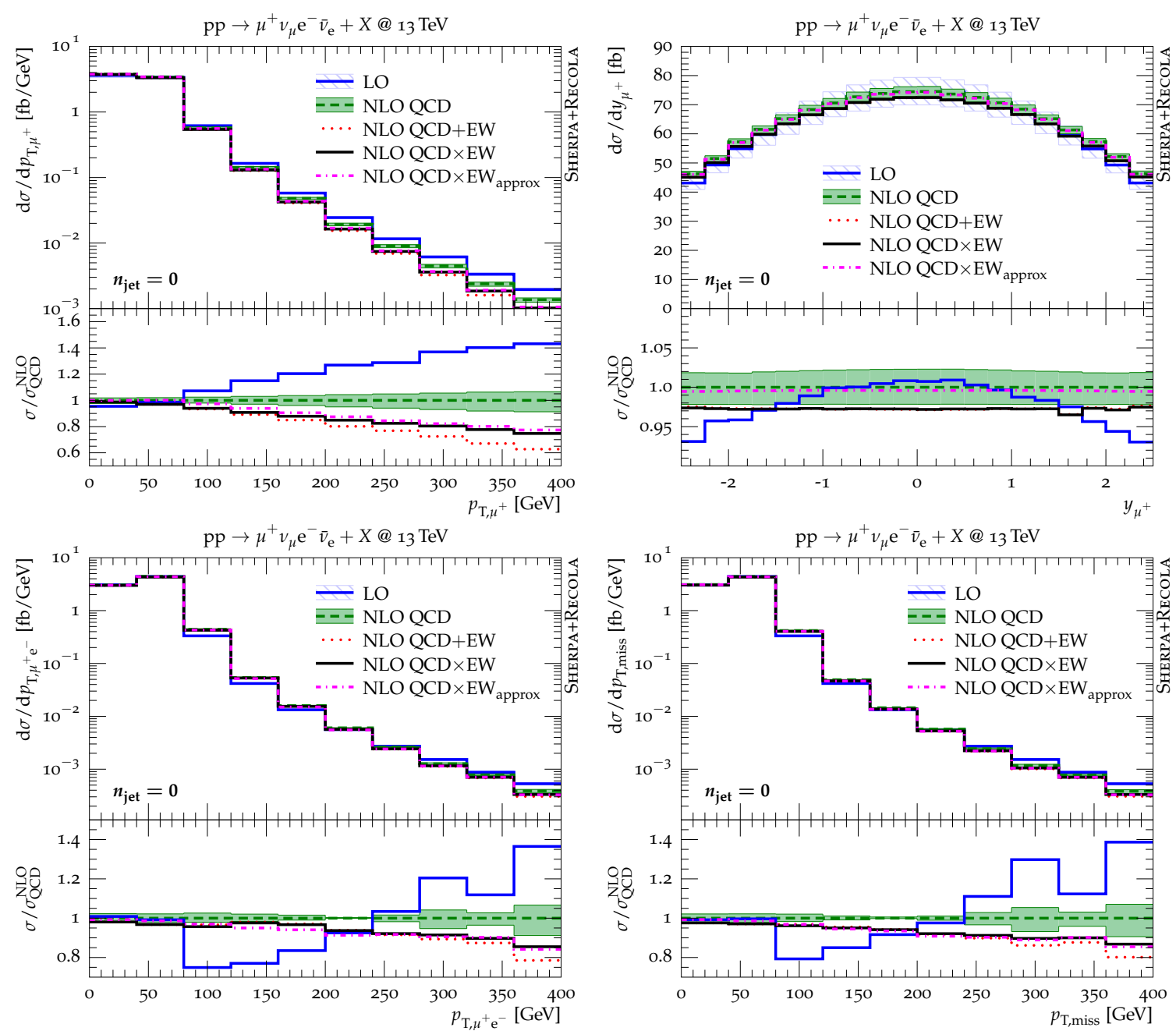

Figure 4. Differential distributions for $\mathrm{pp} \rightarrow \mu^{+} \nu_{\mu} \mathrm{e}^{-} \bar{\nu}_{\mathrm{e}}$ at LO, NLO QCD, NLO QCD + EW, NLO $\mathrm{QCD} \times \mathrm{EW}$, and $\mathrm{NLO} \mathrm{QCD} \times \mathrm{EW}_{\text {approx }}$ : transverse momentum of the anti-muon (top left), rapidity of the anti-muon (top right), transverse momentum of the anti-muon-electron system (bottom left), and missing transverse momentum (bottom right). The upper panels show the absolute predictions, while the lower ones display the ratio of the various predictions with respect to the NLO QCD predictions.

forbids hard jets that would balance the WW system. The fluctuations in the tails of the $p_{\mathrm{T}, \mu^{+} \mathrm{e}^{-}}$and $p_{\mathrm{T}, \mathrm{miss}}$ distributions are of statistical origin.

The NLO QCD $\times \mathrm{EW}_{\text {approx }}$ results follow closely the exact $\mathrm{NLO} \mathrm{QCD} \times \mathrm{EW}$ ones, staying within $3 \%$ of them in the high- $p_{\mathrm{T}}$ regions, despite the presence of a jet veto which is not accounted for in the integrated-out approximate real-emission corrections. For inclusive quantities, like the muon rapidity distribution or the first two bins of the various transversemomentum distributions, they reproduce the exact NLO EW corrections also within $3 \%$. The behaviour of the approximation thus meets the expectations based on its construction.

In figure 5, invariant-mass distributions and angular distributions are displayed. For the distribution in the invariant mass of the two charged leptons, the QCD corrections are 

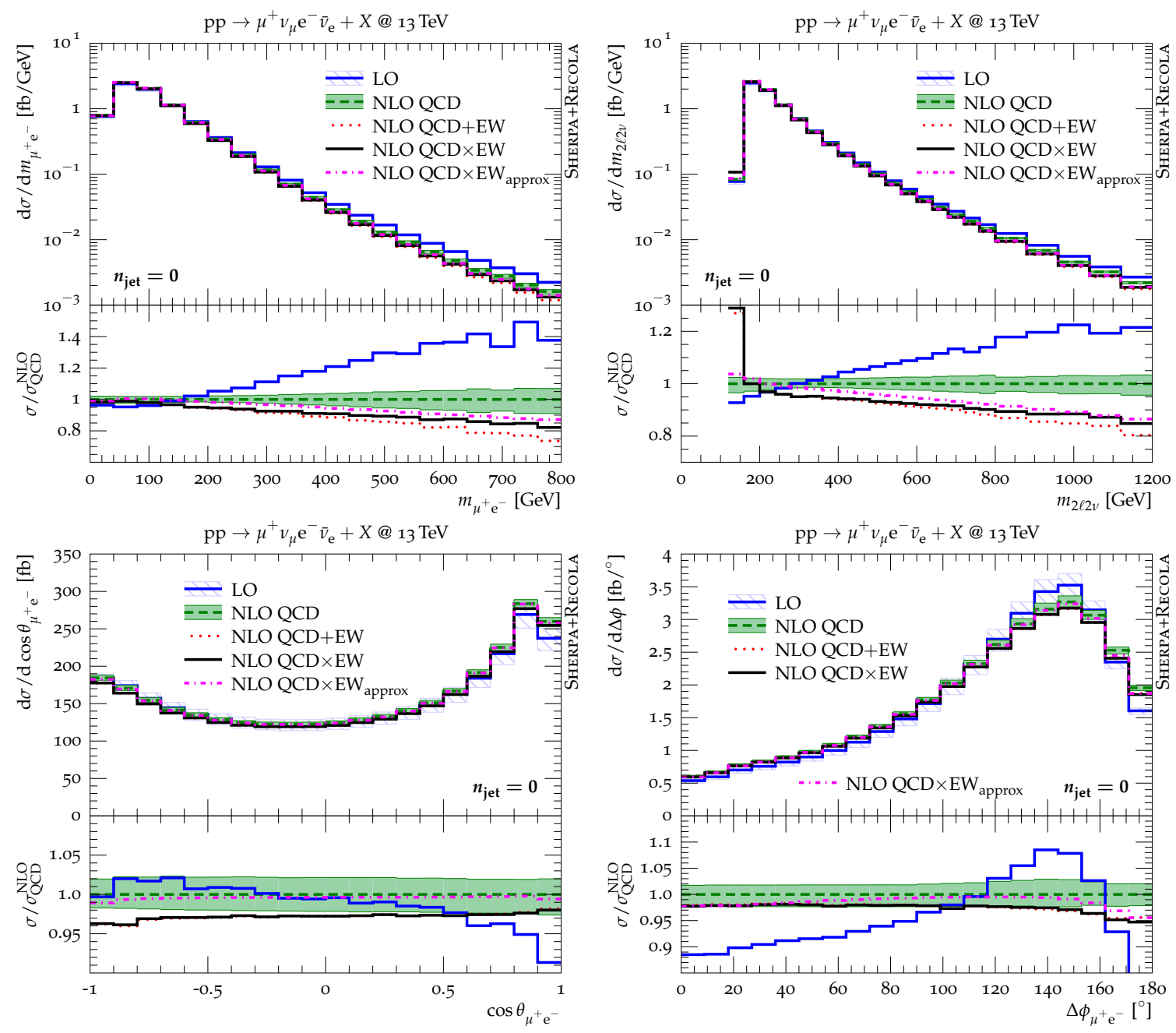

Figure 5. Differential distributions for pp $\rightarrow \mu^{+} \nu_{\mu} \mathrm{e}^{-} \bar{\nu}_{\mathrm{e}}$ at LO, NLO QCD, NLO QCD + EW, $\mathrm{NLO}$ QCD $\times \mathrm{EW}$, and NLO QCD $+\mathrm{EW}_{\text {approx }}$ : invariant mass of the anti-muon and electron (top left), invariant mass of the four leptons (top right), cosine of the angle between the anti-muon and the electron (bottom left), and azimuthal-angle distance between the anti-muon and the electron (bottom right). The upper panels show the absolute predictions, while the lower ones display the ratio of the various predictions with respect to the NLO QCD predictions.

largely negative, increasing towards high invariant mass and reach $-50 \%$ above $0.8 \mathrm{TeV}$. On the other hand, the EW corrections steadily approach $-20 \%$ at $0.8 \mathrm{TeV}$ thanks to the effect of enhanced EW logarithms. Despite not being a physical observable, the distribution in the invariant mass of the four leptons is interesting to study as it serves as a proxy in studies of physics beyond the Standard Model. The behaviour is qualitatively similar to the one of the di-lepton invariant-mass distribution. The distribution in the cosine of the angle between the two charged leptons displays QCD corrections smaller than $1 \%$ near $\theta=\pi$ reaching $+9 \%$ near $\theta=0$. Thus, when the two leptons are back-to-back the QCD corrections are negative, while when they are aligned they turn positive. The latter kinematic situation is the most probable and corresponds to a central production of the two 


\begin{tabular}{|c|c|c|c|c|}
\hline$\sigma^{\mathrm{LO}}[\mathrm{fb}]$ & $\sigma_{\mathrm{QCD}}^{\mathrm{NLO}}[\mathrm{fb}]$ & $\sigma_{\mathrm{EW}}^{\mathrm{NLO}}[\mathrm{fb}]$ & $\sigma_{\mathrm{QCD}+\mathrm{EW}}^{\mathrm{NLO}}[\mathrm{fb}]$ & $\sigma_{\mathrm{QCD} \times \mathrm{EW}}^{\mathrm{NLO}}[\mathrm{fb}]$ \\
\hline $162.5(1)_{-9.1 \%}^{+11.2 \%}$ & $129.5(5)_{-8.9 \%}^{+5.1 \%}$ & $155.5(1)$ & $122.5(5)$ & $123.9(5)$ \\
\hline
\end{tabular}

Table 2. Fiducial cross sections for pp $\rightarrow \mu^{+} \nu_{\mu} \mathrm{e}^{-} \bar{\nu}_{\mathrm{e}} \mathrm{j}$ at $\sqrt{s}=13 \mathrm{TeV}$ at LO, NLO QCD, and NLO EW. Furthermore, results for the additive and multiplicative combination of NLO QCD and NLO EW corrections are given.

gauge bosons. The EW corrections are rather smooth and vary by less than $3 \%$ between the two extreme kinematic configurations. Finally, the distribution in the azimuthal distance between the two charged leptons displays rather moderate QCD corrections. They reach $-10 \%$ around $\Delta \phi_{\mu^{+} \mathrm{e}^{-}} \simeq 140^{\circ}$, i.e. when the two charged leptons are almost in a back-toback configuration. In both angular distributions EW corrections are at the level of a few per cent, however, exceeding the NLO QCD scale-uncertainty estimate.

The approximate NLO EW corrections reproduce the invariant-mass spectra well in the Sudakov regions, though generally slightly worse than the transverse momentum spectra of figure 4. Again, the exact NLO EW corrections for inclusive distributions like $\cos \theta_{\mu^{+} \mathrm{e}^{-}}$ and $\Delta \phi_{\mu^{+} \mathrm{e}^{-}}$are also reproduced within about $3 \%$.

The difference between the additive and multiplicative prescriptions for combining QCD and EW corrections is in general small. However, in regions where both QCD and EW corrections become large, such as for high transverse momenta or invariant masses, the difference can amount to ten per cent or more.

\subsubsection{WWj production}

In the same way as for di-boson production, fiducial cross sections at LO, NLO QCD, and NLO EW accuracy are given for pp $\rightarrow \mu^{+} \nu_{\mu} \mathrm{e}^{-} \bar{\nu}_{\mathrm{ej}}$ in table 2. Notably, QCD scale uncertainties are almost a factor two larger than for WW production owing to the additional power in the strong coupling already at LO. As before, the inclusion of NLO QCD corrections reduces the scale uncertainties observed at LO. In our calculational set-up, the numerical value of the NLO QCD corrections differ significantly from those for the WW channel and amount to $-20 \%$ owing to the strong jet veto. The NLO EW corrections amount to $-4.3 \%$, very similar to the case of $\mathrm{WW}$ production. This could be expected, since the additional gluon does not take part in the EW interaction. As a consequence of the sizeable QCD corrections, the additive and multiplicative combination of QCD and EW corrections differ by about $1 \%$.

As for WW, there exists a loop-induced contribution at order $\mathcal{O}\left(\alpha_{\mathrm{s}}^{3} \alpha^{4}\right)$ from the partonic process gg $\rightarrow \mu^{+} \nu_{\mu} \mathrm{e}^{-} \bar{\nu}_{\mathrm{e}} \mathrm{g}$, which is not included in table 2 . Its fiducial cross section amounts to $11.941(3)_{-27.5 \%}^{+41.3 \%} \mathrm{fb}$, i.e. $7.3 \%$ of the tree-level prediction, which is slightly smaller in comparison to the corresponding contribution to the WW process. However, the scale uncertainty on this channel is particularly large.

In the following, the same set of distributions is shown as for the case of WW production. In addition, we include the distributions in the transverse momentum and rapidity of the hardest jet (ordered in transverse momentum), which are displayed first in figure 6 . 
In the tail of the jet transverse-momentum distribution, very large NLO QCD corrections as well as sizeable NLO EW corrections appear. For the distribution in the rapidity of the jet, the QCD corrections (not visible on the lower panel) can be as large as $-40 \%$. On the other hand, the EW corrections are rather stable over the whole kinematic range. The qualitative behaviour is similar for the distributions in the transverse momentum and the rapidity of the anti-muon. Quantitatively the QCD corrections are smaller for the distribution in the transverse momentum for the anti-muon than in the one for the leading jet, but are at the same level for the rapidity distributions of the anti-muon and leading jet. Finally, the QCD corrections to the distributions in the transverse momentum of the antimuon-electron system and in the missing transverse momentum are rather different from the case of WW production, being much larger at large transverse momenta. The different behaviour just above $M_{\mathrm{W}}$ is due to the fact that for $\mathrm{WWj}$ production configurations with two resonant $\mathrm{W}$ bosons contribute in this phase-space region, while these are excluded for WW production at LO. On the other hand, the QCD corrections to the distributions in $p_{\mathrm{T}, \mu^{+} \mathrm{e}^{-}}$or $p_{\mathrm{T}, \mathrm{miss}}$ are similar to those for the distribution in the transverse momentum of the jet owing to the recoil of the jet against the WW system. The QCD corrections exceed $-100 \%$ above $150 \mathrm{GeV}$ (when normalised to LO, the QCD corrections stay below $100 \%$ in the considered range). This is a consequence of the applied jet veto, which reduces the cross section even stronger in the presence of high- $p_{\mathrm{T}}$ jets. We note that such large jet-veto logarithms can be avoided by adopting a dynamic definition of the jet veto [14, 17]. These can also be efficiently handled by the parton shower and/or merging procedures as shown below. The scale uncertainty grows very large towards high transverse momentum owing to the large NLO QCD contribution and the cancellations between LO and NLO QCD. As a consequence, for all observables considered in figure 6 the NLO EW corrections stay within the NLO QCD scale uncertainty bands.

In figure 7 we present the di-lepton and four-lepton invariant-mass distribution as well as the distributions in the polar and azimuthal separation of the charged leptons. Both invariant-mass distributions receive QCD corrections of about $-80 \%$ for large invariant masses. The EW corrections are more moderate and reach $-20 \%$ in the considered kinematic range, thereby almost exceeding the NLO QCD scale uncertainties. As in the case of WW production, both angular observables do not exhibit enhanced corrections. The QCD corrections (not visible in the lower panels) are essentially flat, and the EW corrections vary by a few per cent only.

The inclusion of approximate NLO EW corrections in the $\mathrm{NLO} \mathrm{QCD} \times \mathrm{EW}_{\text {approx }}$ results again reproduces the exact NLO EW corrections in the same manner as previously observed for the zero-jet case, i.e. within a few per cent.

As a consequence of the very large QCD corrections and sizeable EW corrections for the invariant-mass and, in particular, transverse-momentum distributions, the two prescriptions to combine QCD and EW corrections give rather different predictions at high invariant masses and transverse momenta. The cross section becomes even negative in the additive combination for large transverse momenta. 

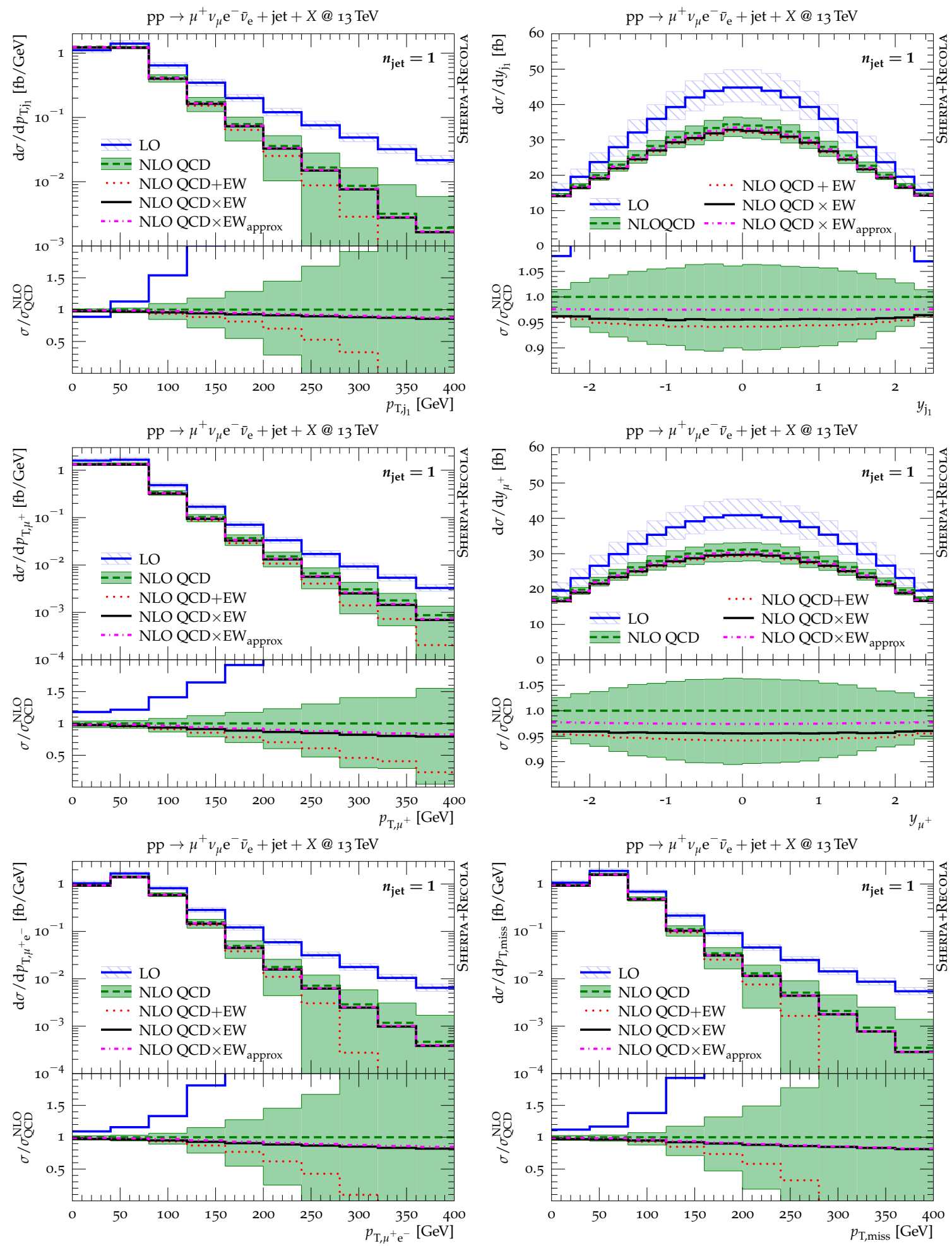

Figure 6. Differential distributions for pp $\rightarrow \mu^{+} \nu_{\mu} \mathrm{e}^{-} \bar{\nu}_{\mathrm{e}} \mathrm{j}$ at LO, NLO QCD, NLO QCD +EW, NLO $\mathrm{QCD} \times \mathrm{EW}$, and $\mathrm{NLO} \mathrm{QCD} \times \mathrm{EW}_{\text {approx }}$ : transverse momentum of the jet (top left), rapidity of the jet (top right), transverse momentum of the anti-muon (middle left), rapidity of the anti-muon (middle right), transverse momentum of the anti-muon-electron system (bottom left), and missing transverse momentum (bottom right). The upper panels show the absolute predictions, while the lower ones display the ratio of the various predictions with respect to the NLO QCD predictions. 

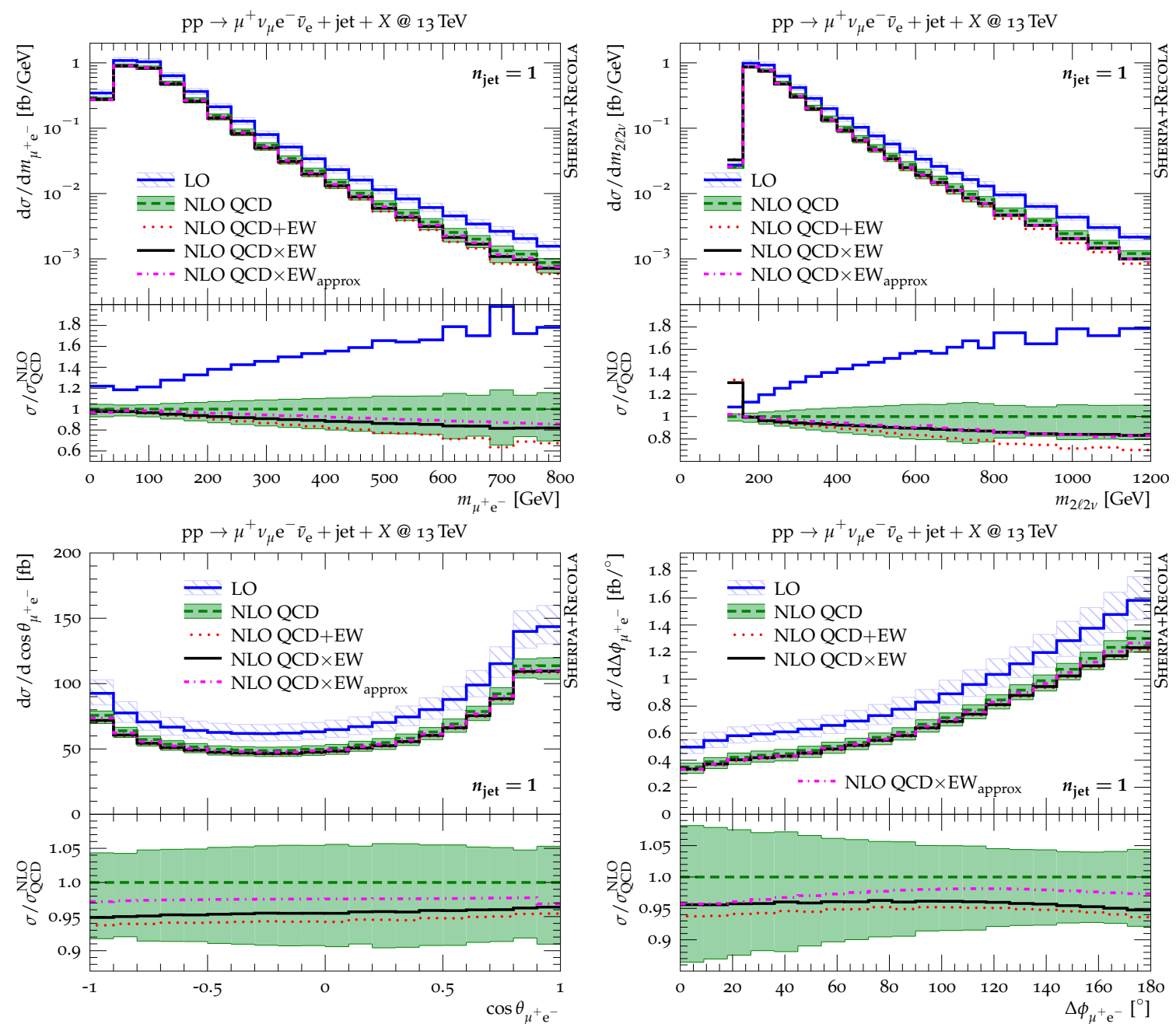

Figure 7. Differential distributions for pp $\rightarrow \mu^{+} \nu_{\mu} \mathrm{e}^{-} \bar{\nu}_{\mathrm{e}} \mathrm{j}$ at LO, NLO QCD, NLO QCD + EW, $\mathrm{NLO}$ QCD $\times \mathrm{EW}$, and $\mathrm{NLO} \mathrm{QCD} \times \mathrm{EW}_{\text {approx }}$ : invariant mass of the anti-muon and electron (top left), invariant mass of the four leptons (top right), cosine of the angle between the anti-muon and the electron (bottom left), and azimuthal-angle distance between the anti-muon and the electron (bottom right). The upper panels show the absolute predictions, while the lower ones display the ratio of the various predictions with respect to the NLO QCD predictions.

\subsubsection{Ratios of $\mathrm{WW}$ and $\mathrm{WWj}$}

In this section, ratios of fiducial cross sections and differential distributions between $\mathrm{pp} \rightarrow$ $\mu^{+} \nu_{\mu} \mathrm{e}^{-} \bar{\nu}_{\mathrm{e}}$ and $\mathrm{pp} \rightarrow \mu^{+} \nu_{\mu} \mathrm{e}^{-} \bar{\nu}_{\mathrm{e}} \mathrm{j}$ are studied. Motivated by the closely related final states of the two processes, the level of universality of the higher-order QCD and EW corrections can be probed. Furthermore, this cross-section ratio has been measured by the ATLAS collaboration in ref. [3] and is expected to have reduced experimental systematic uncertainties. Ratios of fiducial cross sections at LO, NLO QCD and EW as well as their additive and multiplicative combination are compiled in table 3. In these ratios as well as in the ratios of distributions below we always treat the scale uncertainties of $\mathrm{WW}$ and $\mathrm{WWj}$ production as correlated. While the NLO QCD corrections to the ratio amount to $-20 \%$, 


\begin{tabular}{|c|c|c|c|c|}
\hline LO & NLO QCD & NLO EW & NLO QCD + EW & NLO QCD $\times$ EW \\
\hline $0.508_{-13.5 \%}^{+17.5 \%}$ & $0.403_{-6.9 \%}^{+2.9 \%}$ & 0.500 & 0.392 & 0.397 \\
\hline
\end{tabular}

Table 3. Ratios of fiducial cross sections between pp $\rightarrow \mu^{+} \nu_{\mu} \mathrm{e}^{-} \bar{\nu}_{\mathrm{ej}}$ and $\mathrm{pp} \rightarrow \mu^{+} \nu_{\mu} \mathrm{e}^{-} \bar{\nu}_{\mathrm{e}}$ at $\sqrt{s}=13 \mathrm{TeV}$ at LO, NLO QCD, and NLO EW as well as for the additive and multiplicative prescription to combine NLO QCD and NLO EW corrections.

the EW corrections yield $-1.5 \%$ only. As a consequence, the additive and multiplicative prescriptions for the combination of QCD and EW corrections agree within 1.3\%, which is basically the difference observed for the WWj cross section. We furthermore note, that the scale uncertainty on the cross-section ratio (with fully correlated scale uncertainties for $\mathrm{WW}$ and $\mathrm{WWj}$ ) significantly reduces when including the NLO QCD corrections.

Next, we show ratios for those differential distributions that have already been discussed for the process pp $\rightarrow \mu^{+} \nu_{\mu} \mathrm{e}^{-} \bar{\nu}_{\mathrm{e}}$ in section 3.1.1. In the upper panels, the ratios

$$
R_{0 \mathrm{j}}^{1 \mathrm{j}}(x)=\frac{\frac{\mathrm{d} \sigma}{\mathrm{d} x}\left(\mathrm{pp} \rightarrow \mu^{+} \nu_{\mu} \mathrm{e}^{-} \bar{\nu}_{\mathrm{e}} \mathrm{j}\right)}{\frac{\mathrm{d} \sigma}{\mathrm{d} x}\left(\mathrm{pp} \rightarrow \mu^{+} \nu_{\mu} \mathrm{e}^{-} \bar{\nu}_{\mathrm{e}}\right)}
$$

are displayed at LO, NLO QCD, NLO QCD $+\mathrm{EW}$, and NLO QCD $\times$ EW accuracy. As before, the approximate $\mathrm{NLO} \mathrm{QCD} \times \mathrm{EW}_{\text {approx }}$ results are added to gauge the quality of the approximation before it is employed in the construction of the multi-jet merged results of section 3.2.3. In the lower panels, these ratios are again normalised to the respective NLO QCD prediction.

In figure 8, the ratios for the transverse-momentum and rapidity distribution of the anti-muon are shown. For the transverse-momentum distribution, NLO QCD corrections are very large and stabilise the ratio towards high transverse momenta. At LO the jet veto (2.21) just affects the pp $\rightarrow \mu^{+} \nu_{\mu} \mathrm{e}^{-} \bar{\nu}_{\mathrm{e}} \mathrm{j}$ channel, only from NLO on it is active for the $\mathrm{pp} \rightarrow \mu^{+} \nu_{\mu} \mathrm{e}^{-} \bar{\nu}_{\mathrm{e}}$ process. The two processes receive NLO corrections of rather different size providing the observed stabilisation in terms of smaller higher-order corrections in the ratios. The two prescriptions to combine QCD and EW corrections behave rather differently. While the additive combination differs considerably from the pure QCD result for $p_{\mathrm{T}, \mu^{+}}>200 \mathrm{GeV}$, the multiplicative one stays close to it. This is in agreement with the observation that the leading-logarithmic corrections for the two processes $\mathrm{WW}$ and WWj are strongly correlated. In fact, these are related to the EW charges of the external lines, which are the same for both processes. This can be deduced from the general results on the leading one-loop EW corrections presented in ref. [87] and from the results based on soft-collinear effective theory in refs. $[88,89]$. This is a strong motivation to prefer the multiplicative prescription. The difference between the two prescriptions should not be taken as an uncertainty. Moreover, it supports the merging approach presented in section 2.4 which rests on the assumption that leading EW corrections are rather similar for different final-state jet multiplicities. While the anti-muon rapidity distribution does not exhibit a strong difference between the two combinations over the whole phase space, the ratio for the multiplicative combination is closer to the pure QCD result and less dependent on the rapidity. 

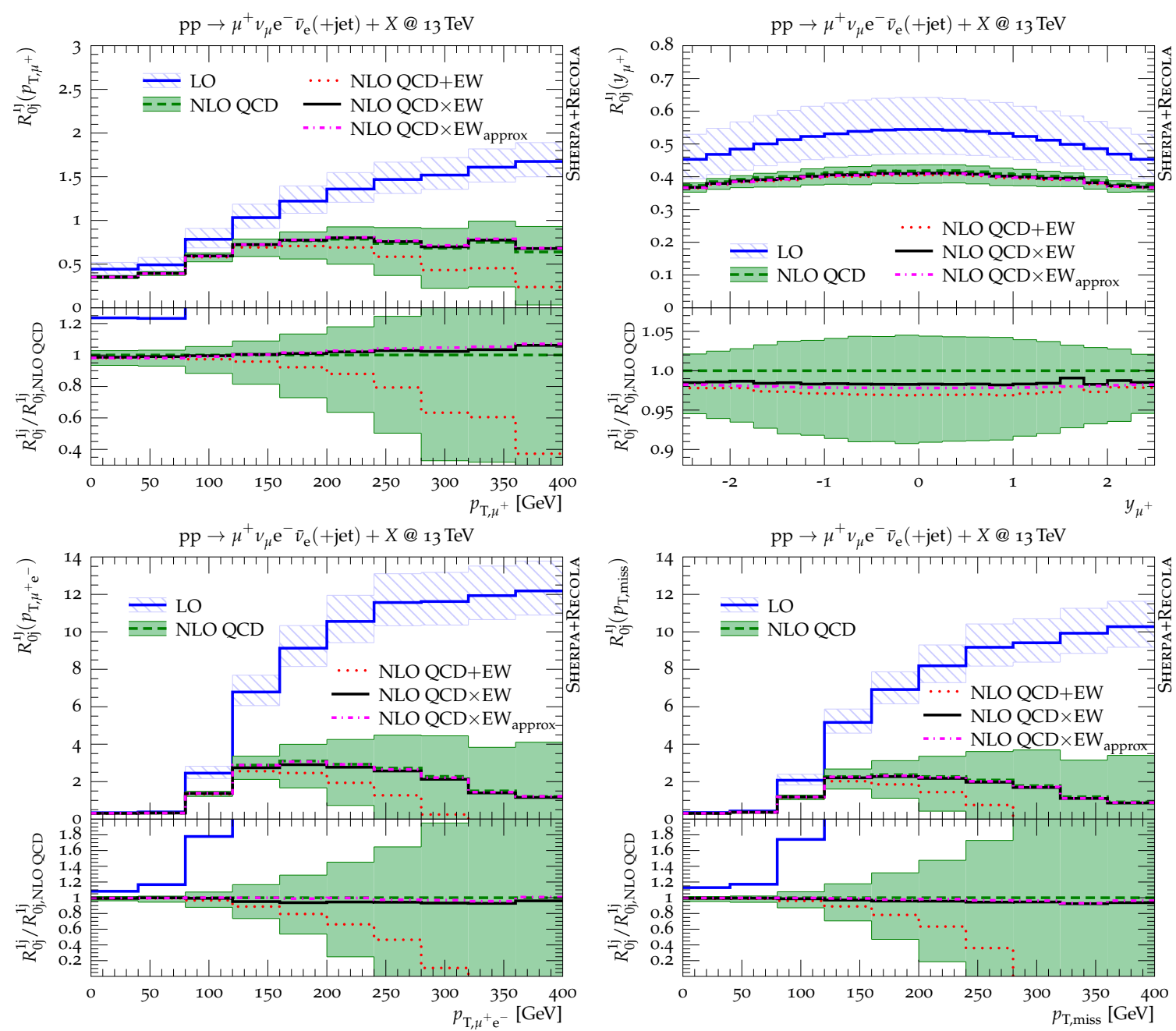

Figure 8. Ratios of differential distributions between pp $\rightarrow \mu^{+} \nu_{\mu} \mathrm{e}^{-} \bar{\nu}_{\mathrm{e}} \mathrm{j}$ and pp $\rightarrow \mu^{+} \nu_{\mu} \mathrm{e}^{-} \bar{\nu}_{\mathrm{e}}$ at LO, $\mathrm{NLO} \mathrm{QCD}, \mathrm{NLO} \mathrm{QCD}+\mathrm{EW}, \mathrm{NLO} \mathrm{QCD} \times \mathrm{EW}$, and $\mathrm{NLO} \mathrm{QCD} \times \mathrm{EW}_{\text {approx }}$ : transverse momentum of the anti-muon (top left), rapidity of the anti-muon (top right), transverse momentum of the antimuon-electron system (bottom left), and missing transverse momentum (bottom right). The upper panels show the absolute predictions, while the lower ones display the ratio between the various predictions and the respective NLO QCD result.

The ratios of the distributions in the transverse momentum of the two charged leptons and the missing energy show very large variations. They are particularly sensitive to the applied jet veto, which is adequately accounted for at NLO QCD only, in particular for the $\mathrm{WW}$ channel. Indeed, at $\mathrm{LO}$ the $\mathrm{WW}$ and $\mathrm{WWj}$ processes have rather different kinematics. With the inclusion of real radiations the descriptions of both processes become closer, and the ratios stabilise in terms of smaller higher-order corrections.

The ratios for the invariant-mass and angular distributions shown in figure 9 confirm the trend seen in the other distributions. When using the multiplicative prescription to include the EW corrections on top of the QCD corrections, the ratios depend only very weakly on the invariant masses and angles, while for the additive combination this depen- 

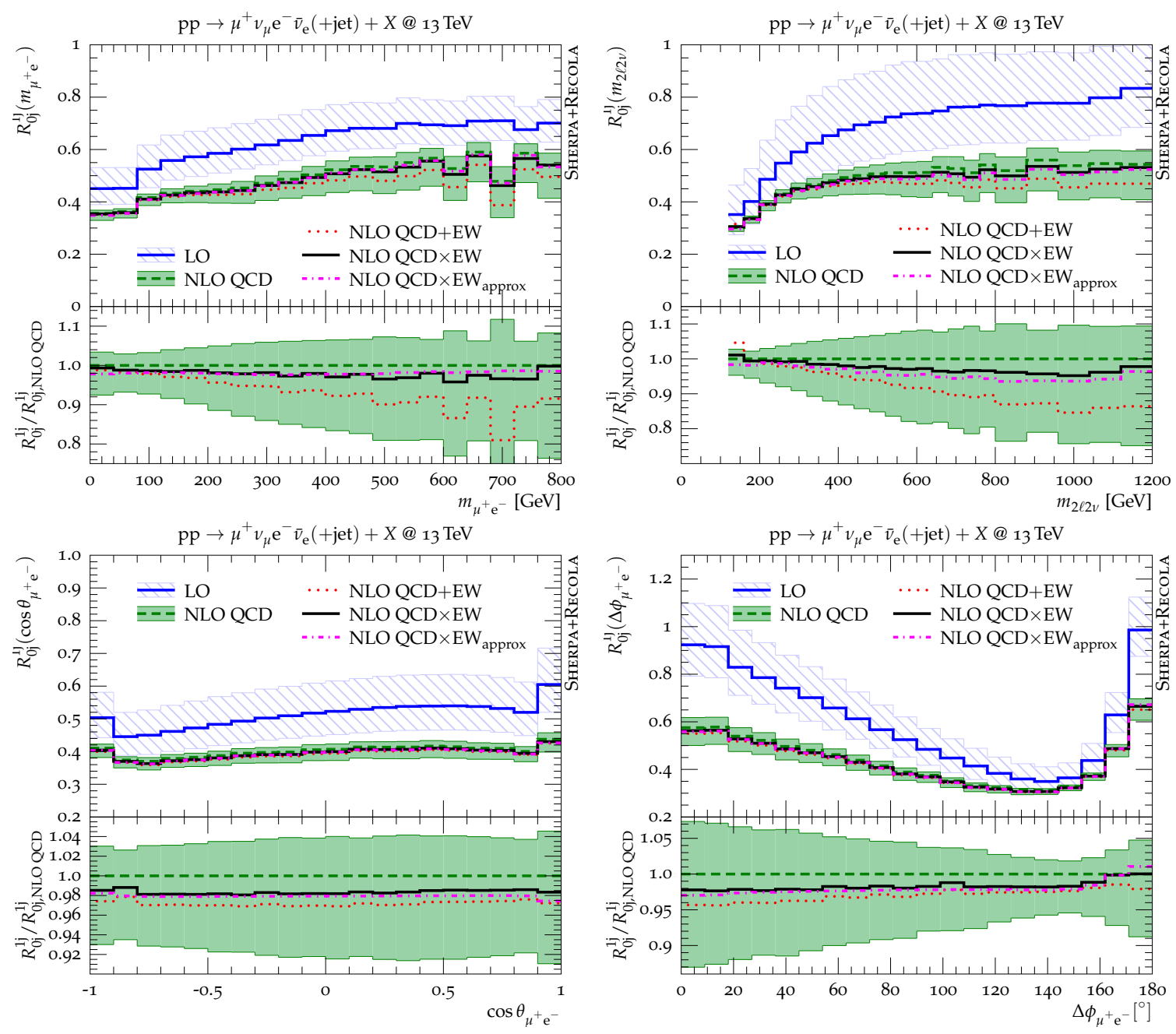

Figure 9. Ratios of differential distributions between pp $\rightarrow \mu^{+} \nu_{\mu} \mathrm{e}^{-} \bar{\nu}_{\mathrm{e}} \mathrm{j}$ and $\mathrm{pp} \rightarrow \mu^{+} \nu_{\mu} \mathrm{e}^{-} \bar{\nu}_{\mathrm{e}}$ at $\mathrm{LO}, \mathrm{NLO} \mathrm{QCD}, \mathrm{NLO} \mathrm{QCD}+\mathrm{EW}, \mathrm{NLO} \mathrm{QCD} \times \mathrm{EW}$, and $\mathrm{NLO} \mathrm{QCD} \times \mathrm{EW}_{\text {approx }}$ : invariant mass of the anti-muon and electron (top left), invariant mass of the four leptons (top right), cosine of the angle between the anti-muon and the electron (bottom left), and azimuthal-angle distance between the anti-muon and the electron (bottom right). The upper panels show the absolute predictions, while the lower ones display the ratio between the various predictions and the respective NLO QCD result.

dence is more pronounced. The more or less decent behaviour of the additive prescription, in particular for the angular distributions, is due to the smallness of the corresponding QCD and EW corrections.

As for the individual cross sections, the approximate NLO EW corrections reproduce the exact cross-section ratios well in the Sudakov region, for which they were constructed. Interestingly, and to some extent accidentally, the exact results for the ratios of inclusive observables like the muon rapidity, $\cos \theta_{\mu^{+} e^{-}}$and $\Delta \phi_{\mu^{+} e^{-}}$are also found to be very well reproduced and in particular better than for the individual cross sections. This can be qualitatively understood by the fact that the main feature of the virtual EW approximation is 
the crude and inclusive approximation of real emission corrections via the Catani-Seymour $I$-operator. Since a large part of the missing effects concerns the final-state leptons, these real emission corrections are to a certain degree universal for the different jet multiplicities and thus cancel to some extent in the ratios.

The above conclusion that the multiplicative prescription is preferred applies for the set-up considered here. In general, such a statement should be checked on a case-by-case basis for different phase spaces. Nonetheless, as the argument relies on factorisation in the bulk of the cross section, the multiplicative approach should also be preferred for set-ups (possibly with a slightly different jet veto) that share a large portion of the phase space.

\subsection{Multi-jet merged results}

In this section we present predictions based on the merging of the NLO QCD matrix elements for $\mathrm{pp} \rightarrow \mu^{+} \nu_{\mu} \mathrm{e}^{-} \bar{\nu}_{\mathrm{e}}+0,1 \mathrm{j}$ and tree-level matrix elements for $\mathrm{pp} \rightarrow \mu^{+} \nu_{\mu} \mathrm{e}^{-} \bar{\nu}_{\mathrm{e}}+2,3 \mathrm{j}$, each matched to the SHERPA Catani-Seymour parton shower. We implement approximate NLO EW corrections in an additive or multiplicative manner for the zero- and one-jet matrix elements. However, these also enter the higher-multiplicity tree-level processes through a local $K$ factor (cf. section 2.4). By merging parton-shower matched matrix elements of varying final-state parton multiplicity we arrive at a fully inclusive event sample for WW production. This sample can then be analysed for the zero- and one-jet selection criteria, without the need to perform dedicated calculations, as it is the case for the NLO fixed-order predictions. Furthermore, the inclusion of higher-multiplicity processes and parton-shower resummation accounts for possible higher jet multiplicities and in turn provides a more adequate description of the jet-veto conditions applied. In the following we neglect effects from the parton-to-hadron transition, as well as underlying-event contributions appearing in hadron collisions, which allows us to directly compare fixed-order calculations with perturbative parton-shower Monte Carlo predictions.

\subsubsection{Fixed-order vs. merged results at NLO QCD}

We begin the discussion by comparing MEPs@NLO QCD predictions from SHERPA for the zero- and one-jet exclusive event selections against the fixed-order NLO QCD results presented in section 3.1. Therefore, in the merged predictions for both multiplicities, further jets are vetoed. To this end, we present predictions following the default CKKW scale-setting prescription as outlined in section 2.4. Accordingly, for each hard-partonic event configuration a clustering algorithm is applied to reconstruct the kinematics of the corresponding pp $\rightarrow \mu^{+} \nu_{\mu} \mathrm{e}^{-} \bar{\nu}_{\mathrm{e}}$ core process. This clustering procedure defines the CKKW scale $\mu_{\mathrm{CKKW}}$ through the reconstructed parton branching scales, cf. eq. (2.4), as well as the core scale $\mu_{\text {core }}$ defined on the arrived-at core process. In turn, $\mu_{\text {core }}$ then determines $\mu_{\mathrm{F}}$ and $\mu_{\mathrm{Q}}$ through eq. (2.5). Through this procedure both the fixed-order accuracies of the matrix-element calculations and the resummation accuracy of the parton shower are preserved.

Furthermore, we present results based on an alternative scale-setting prescription, dubbed proto-merging, where for each hard-parton configuration we use

$$
\mu_{\mathrm{R}}=\mu_{\mathrm{F}}=\mu_{\mathrm{Q}}=\frac{1}{2} \bar{E}_{\mathrm{T}, \mathrm{W}}
$$


of the $n$-jet process without clustering any partons first, i.e. we set all three scales equal to the scales used in the corresponding fixed-order calculation without reconstructing emission scales or a core process. While this respects the fixed-order NLO accuracy of each partonic subsample, it spoils the resummation property of the QCD parton shower. ${ }^{5}$ It thus cannot be considered a consistently merged MEPs description, but is included in the following comparison for illustrative purposes.

By invoking the parton shower we include all-order corrections to the inclusive $\mathrm{pp} \rightarrow$ $\mu^{+} \nu_{\mu} \mathrm{e}^{-} \bar{\nu}_{\mathrm{e}}$ production process and, as a consequence, the jet veto affects also the zero- and one-jet selections. As we focus on QCD corrections, we do not include QED corrections due to soft-photon emission [57] or EW effects at this stage.

To estimate the dominant theoretical uncertainties we consistently vary the renormalisation and factorisation scales in the matrix-element and parton-shower components [86, 90]. As before, we consider the 7-point variations of the two scales $\mu_{\mathrm{R}}$ and $\mu_{\mathrm{F}}$ by factors of $\frac{1}{2}$ and 2 . We do not assess the systematics associated with the choice of the merging parameter $Q_{\text {cut }}$ as well as the resummation scale $\mu_{\mathrm{Q}}$ as these can be expected to be of smaller size [24, 27, 39, 91-93].

In the following plots we compare the NLO QCD fixed-order results with MEPs@NLO QCD predictions for two different scale choices. In the upper panels we show absolute predictions at NLO QCD accuracy as well as with MEPS@NLO parton-shower matching for the default CKKW scale setting (2.4) and (2.5) as well as for the scale setting (3.6) corresponding to the fixed-order results. The lower panels show the corresponding results normalised to the NLO QCD ones. Scale uncertainties are indicated by the envelopes of the bands and should be understood as an order of magnitude estimate of missing higher-order corrections.

WW production. In figures 10 and 11 we compile the set of exclusive zero-jet observables for the process pp $\rightarrow \mu^{+} \nu_{\mu} \mathrm{e}^{-} \bar{\nu}_{\mathrm{e}}$ studied in section 3.1.1 already. We begin by considering the anti-muon transverse-momentum distribution in figure 10. The two MEPs@NLO QCD predictions agree rather well with the NLO QCD result in the bulk of the distribution. In the tail of the distribution, which is suppressed by three orders of magnitudes, the two merged predictions are on the edge of the scale-uncertainty band of the fixed-order prediction. For low to intermediate transverse momenta the uncertainty of the proto-merged prediction reproduces well the fixed-order uncertainty. However, for larger $p_{\mathrm{T}, \mu^{+}}$it is significantly reduced, as the MEPs@NLO method accounts for a proper

${ }^{5}$ This can be seen by considering two effects:

1. The shower starting scale $\mu_{\mathrm{Q}}$ will not be set to the scale of the reconstructed core process for all processes with at least one parton in the final state. This existing parton will then not be correctly embedded in the parton-shower evolution of this core process. Since $\mu_{\mathrm{Q}}$ will be typically higher than when using a proper merging procedure, the Sudakov vetoes generated will be too large.

2. The scale of the strong coupling associated with the emission of a parton needs to be set to the relative transverse momentum with respect to its reconstructed emitter parton in order to recover the logarithms produced by the parton shower. Since here a global scale is used which typically is larger than its nodal value, the resulting strong coupling will be too small. 

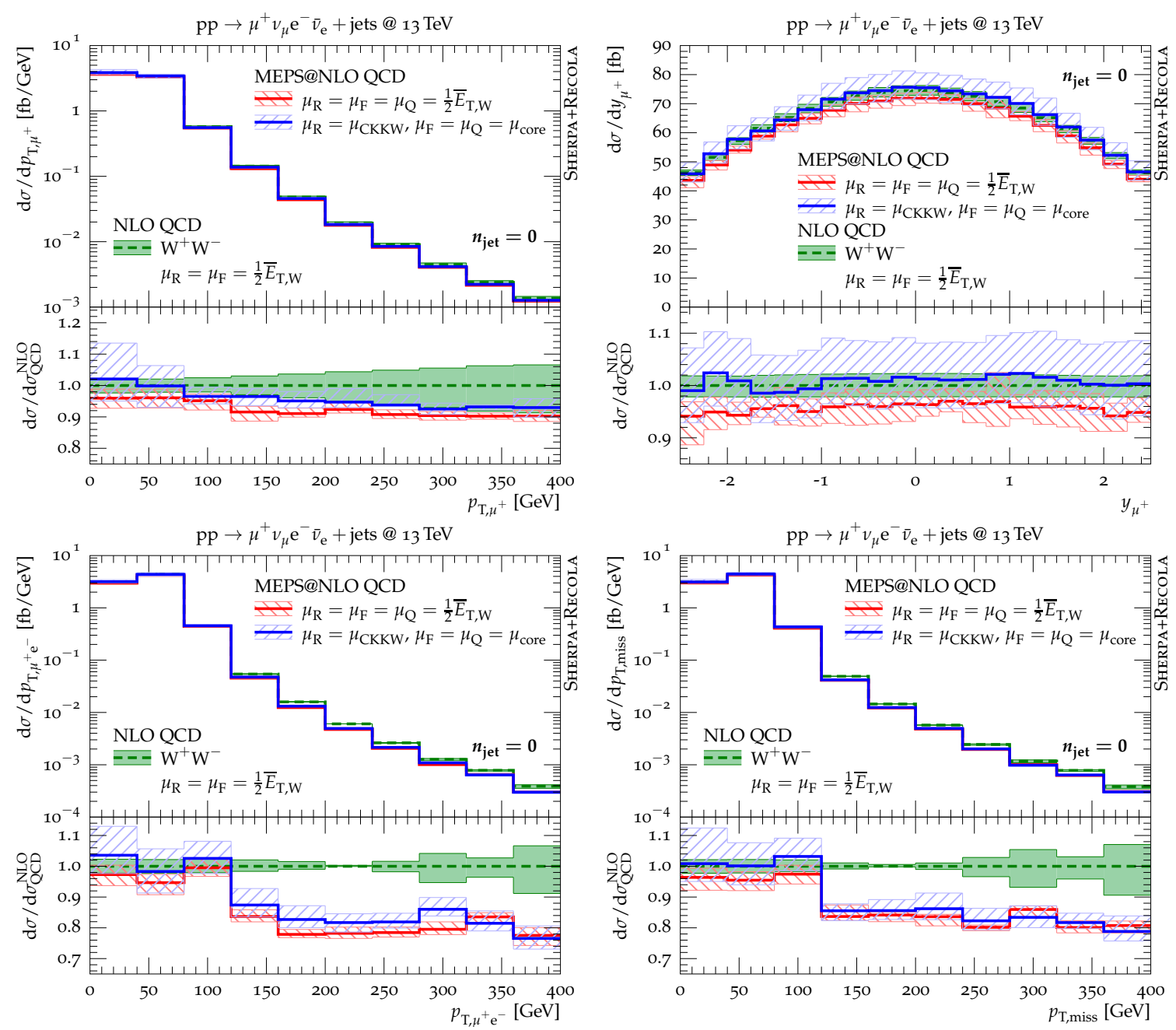

Figure 10. Comparison of NLO QCD fixed-order results for the process pp $\rightarrow \mu^{+} \nu_{\mu} \mathrm{e}^{-} \bar{\nu}_{\mathrm{e}}$ with proto-merged and the fully multi-jet merged MEPs@NLO QCD predictions in the $n_{\text {jet }}=0$ event selection: transverse momentum of the anti-muon (top left), rapidity of the anti-muon (top right), transverse momentum of the anti-muon-electron system (bottom left), and missing transverse momentum (bottom right). No QED or EW corrections are taken into account here.

resummation of higher-order QCD corrections. For the default fully multi-jet merged prediction this effect is also observed. However, at lower transverse momenta the uncertainty increases, due to the typically smaller value of the renormalisation scale, determined by the emission scale of the associated partons. From the anti-muon rapidity distribution one can read off that the central production rate predicted by the default CKKW scale-setting prescription is in fact closer to the fixed-order cross section, whereas it features a larger scale uncertainty of about $\pm 8 \%$, compared to only $\pm 2 \%$ of the fixed-order result. While the proto-merged prediction exhibits an uncertainty more closely resembling the fixed-order estimate, its central cross section is reduced by about $4 \%$.

For the distributions in the transverse momentum of the charged-lepton pair and the missing transverse momentum the differences in the theoretical predictions are much more 

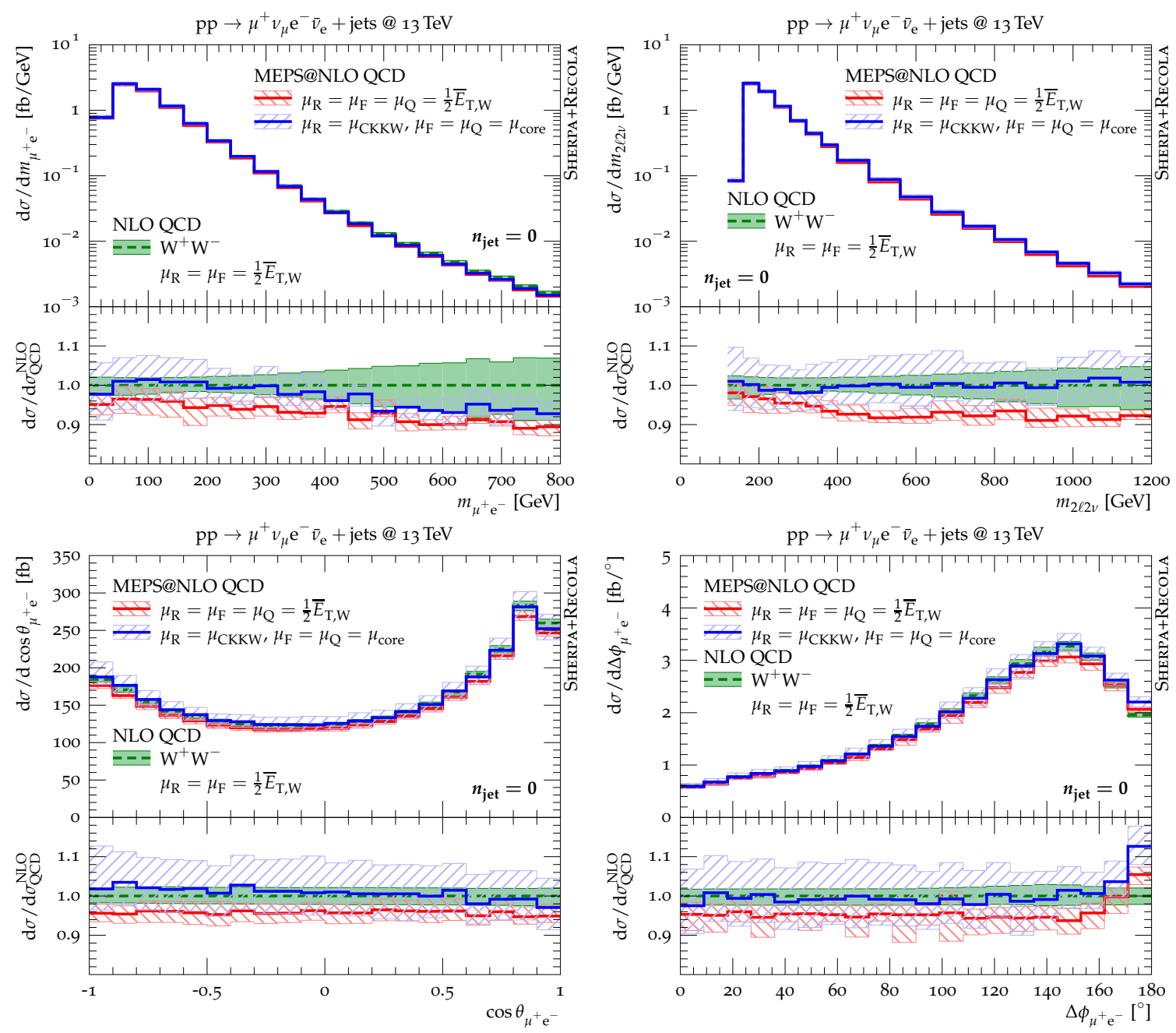

Figure 11. Comparison of NLO QCD fixed-order results for the process pp $\rightarrow \mu^{+} \nu_{\mu} \mathrm{e}^{-} \bar{\nu}_{\mathrm{e}}$ with proto-merged and the fully multi-jet merged MEPs@NLO QCD predictions in the $n_{\text {jet }}=0$ event selection: invariant mass of the anti-muon and electron (top left), invariant mass of the four leptons (top right), cosine of the angle between the anti-muon and the electron (bottom left), and azimuthalangle distance between the anti-muon and the electron (bottom right). No QED or EW corrections are taken into account here.

sizeable. Up to about the $\mathrm{W}$-boson mass the merged predictions agree well with the fixed-order result, however, they predict significantly smaller event rates beyond $M_{\mathrm{W}}+$ $p_{\mathrm{T}, \mathrm{j}, \mathrm{cut}}$. This originates from the same reason as in the fixed-order case namely that, due to kinematic constraints, the inclusion of real radiations beyond the $\mathrm{W}$-boson mass lifts the cross section. In addition, above this threshold the jet-veto criterion plays a significant role as in the NLO QCD calculation of $\mathrm{pp} \rightarrow \mu^{+} \nu_{\mu} \mathrm{e}^{-} \bar{\nu}_{\mathrm{e}}$ it is addressed only at LO accuracy. In the merged calculations, however, the jet veto is modelled by the NLO QCD pp $\rightarrow \mu^{+} \nu_{\mu} \mathrm{e}^{-} \bar{\nu}_{\mathrm{ej}}$ calculation dressed with the parton-shower resummation, leading to a more realistic description of this event-selection criterion. In particular, at high transverse momenta an increased QCD activity is expected that in consequence triggers the jet veto thereby reducing the cross section. 
For the observables depicted in figure 11 the observed pattern is further confirmed. The default multi-jet merged predictions agree nicely with the fixed-order results, with an increased systematic uncertainty in the bulk. However, in particular in the region of high dilepton invariant mass the uncertainty is indeed sizeably reduced with respect to the one of the fixed-order computation. For the angular separation between the two charged leptons the region of $\Delta \phi_{\mu^{+} \mathrm{e}^{-}} \approx \pi$ is affected by the parton-shower resummation. This originates from the suppression of the LO for WW production in this bin (cf. figure 5). As a consequence, the CKKW prescription differs by more than $10 \%$ from the fixed-order prediction.

WWj production. In figures 12 and 13 we present MEPs@NLO QCD predictions for the exclusive one-jet selection for the process pp $\rightarrow \mu^{+} \nu_{\mu} \mathrm{e}^{-} \bar{\nu}_{\mathrm{e}} \mathrm{j}$, studied at NLO QCD in section 3.1.2 already. Most notably, a significant reduction of the systematic uncertainty in phase-space regions affected by the jet-veto criterion is observed as a consequence of including parton-shower resummation. In particular the various transverse-momentum distributions, i.e. $p_{\mathrm{T}, \mathrm{j}_{1}}, p_{\mathrm{T}, \mu^{+}}, p_{\mathrm{T}, \mu^{+} \mathrm{e}^{-}}$, and $p_{\mathrm{T}, \mathrm{miss}}$, receive huge corrections from the inclusion of multiple-emission effects through the parton shower and the higher-multiplicity matrix elements. This results in significantly harder $p_{\mathrm{T}}$ spectra, as through multiple emissions larger recoil can be achieved without triggering the applied jet veto. The inclusion of parton-shower effects and merging significantly increases the predictions which thus do not feature the large negative corrections seen in the fixed-order case that are associated with the jet veto. For the $p_{\mathrm{T}, \mu^{+} \mathrm{e}^{-}}$and $p_{\mathrm{T} \text {,miss }}$ distributions the difference with respect to the NLO QCD prediction gets as large as a factor of 4 . It is to note, however, that these sizeable differences are still compatible with the fixed-order predictions' scale-uncertainty estimates. In contrast, for the merged predictions the uncertainties remain at the $10 \%$ level.

From the jet and anti-muon rapidity distributions in figure 12 it is apparent that the total production rate obtained for the default CKKW scale-setting prescription used in the fully multi-jet merged MEPs@NLO calculation is in very good agreement with the fixedorder NLO QCD result. In fact, for these rather inclusive observables these two central predictions almost coincide, while the proto-merged prediction, using the scale $\mu_{\mathrm{R}}=\mu_{\mathrm{F}}=$ $\mu_{\mathrm{Q}}=\frac{1}{2} \bar{E}_{\mathrm{T}, \mathrm{W}}$ throughout shows a small shape distortion. However, as observed for the zero-jet process already, the estimated systematic uncertainty of the fully merged sample is somewhat increased with respect to the proto-merged and the fixed-order calculation.

In figure 13 the $\cos \theta_{\mu^{+} \mathrm{e}^{-}}$distribution confirms this pattern. For the charged-leptons' polar-angle separation, however, a small enhancement towards smaller values of $\Delta \phi_{\mu^{+} \mathrm{e}^{-}}$ can be observed in the merged predictions. For the two invariant-mass distributions a significant reduction of the scale uncertainty in the high-mass regions is observed. The merged sample tends to populate these phase-space regions somewhat more, though the central predictions stay mainly within the uncertainty band of the fixed-order result.

\subsubsection{Including EW corrections via the virtual approximation}

Having compared the MEPs@NLO QCD predictions against the fixed-order calculations for the zero- and one-jet selection, we now progress by considering the inclusion of approximate 

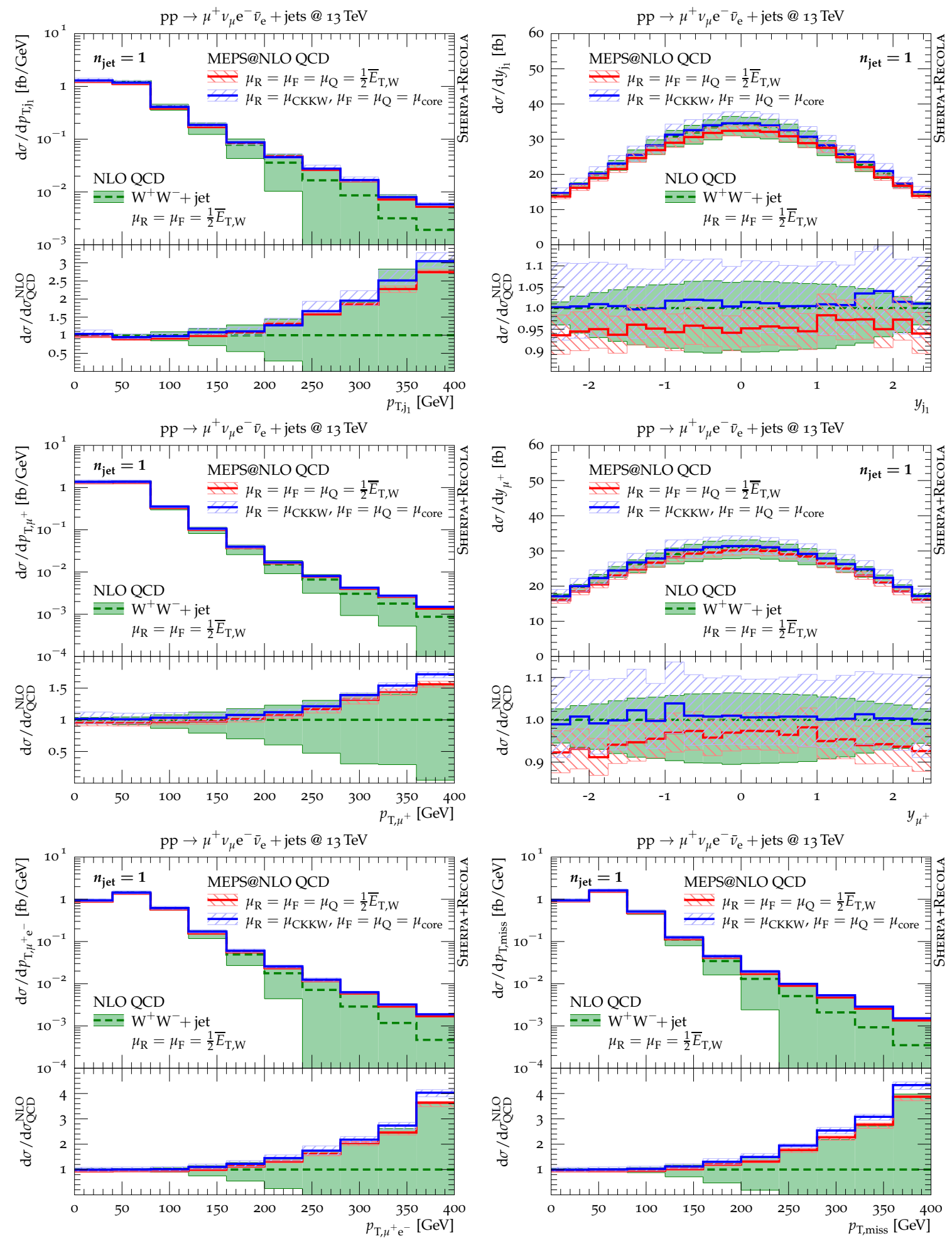

Figure 12. Comparison of NLO QCD fixed-order results for pp $\rightarrow \mu^{+} \nu_{\mu} \mathrm{e}^{-} \bar{\nu}_{\mathrm{e}} \mathrm{j}$ with proto-merged and the fully multi-jet merged MEPs@NLO QCD predictions in the $n_{\text {jet }}=1$ event selection: transverse momentum of the jet (top left), rapidity of the jet (top right), transverse momentum of the anti-muon (middle left), rapidity of the anti-muon (middle right), transverse momentum of the anti-muon-electron system (bottom left), and missing transverse momentum (bottom right). No QED or EW corrections are taken into account here. 

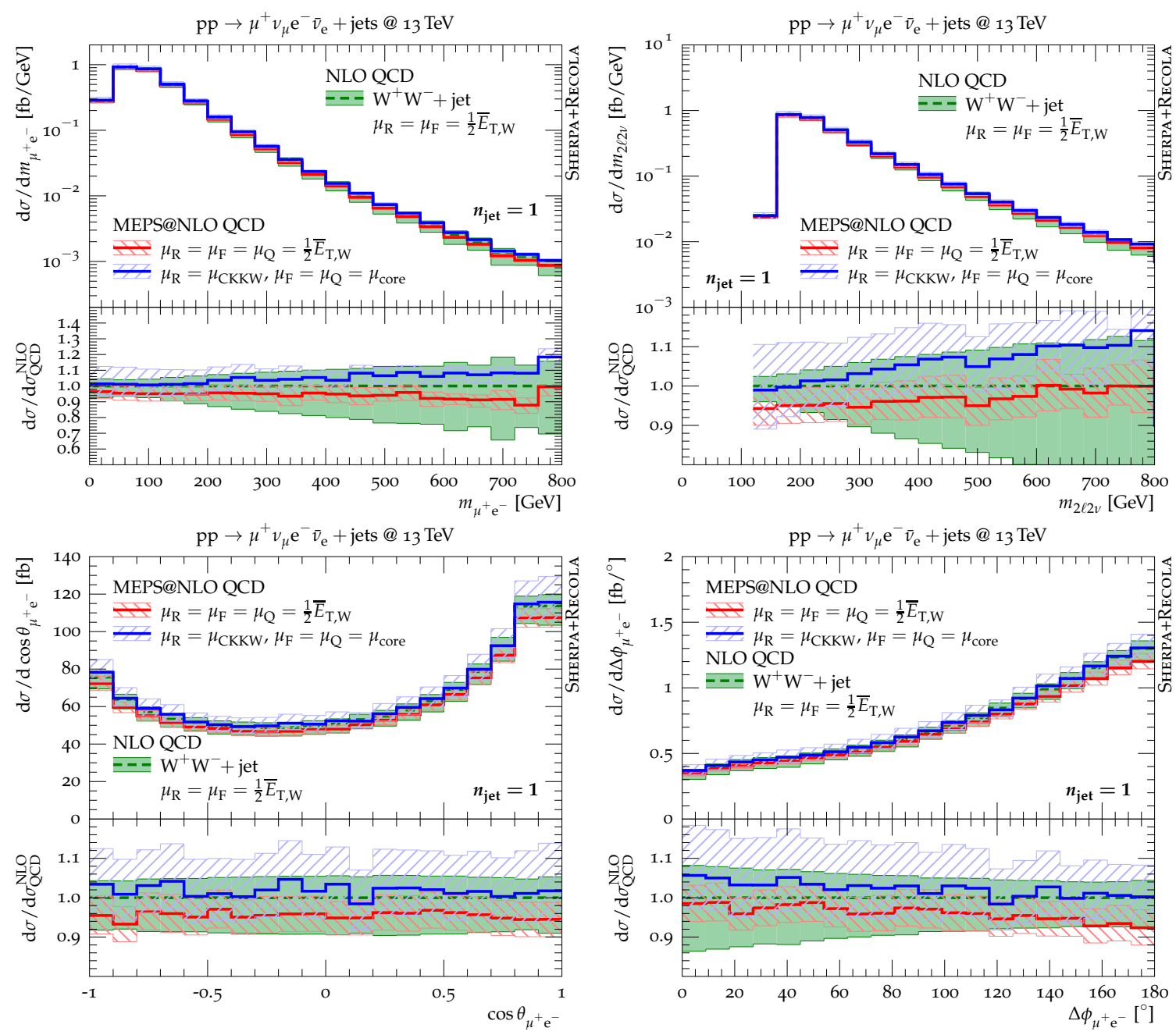

Figure 13. Comparison of NLO QCD fixed-order results for the process pp $\rightarrow \mu^{+} \nu_{\mu} \mathrm{e}^{-} \bar{\nu}_{\mathrm{e}} \mathrm{j}$ with proto-merged and the fully multi-jet merged MEPs@NLO QCD predictions in the $n_{\text {jet }}=1$ event selection: invariant mass of the anti-muon and electron (top left), invariant mass of the four leptons (top right), cosine of the angle between the anti-muon and the electron (bottom left), and azimuthalangle distance between the anti-muon and the electron (bottom right). No QED or EW corrections are taken into account here.

NLO EW corrections into the merged calculations. The accuracy of this approximation has been examined for the different observables under consideration in this paper in section 3.1. The central prediction is formed by the fully multi-jet merged sample based of the NLO QCD matrix elements for pp $\rightarrow \mu^{+} \nu_{\mu} \mathrm{e}^{-} \bar{\nu}_{\mathrm{e}}$ and pp $\rightarrow \mu^{+} \nu_{\mu} \mathrm{e}^{-} \bar{\nu}_{\mathrm{e}} \mathrm{j}$ and the tree-level ones for pp $\rightarrow \mu^{+} \nu_{\mu} \mathrm{e}^{-} \bar{\nu}_{\mathrm{ejj}}$ and $\mathrm{pp} \rightarrow \mu^{+} \nu_{\mu} \mathrm{e}^{-} \bar{\nu}_{\mathrm{e}} \mathrm{jjj}$, all matched to the SHERPA Catani-Seymour dipole shower.

For all matrix-element multiplicities we now include soft-photon resummation effects via the YFS approach. For the zero- and one-jet one-loop matrix elements we furthermore employ the EW virtual approximation, described in section 2.4, both in the additive and multiplicative manner, cf. eqs. (2.11) and (2.12), respectively. We would like to remind the 


\begin{tabular}{|c|c|c|c|}
\hline MePs@Lo & \multicolumn{3}{|c|}{ MePs@NlO } \\
\hline QCD $[\mathrm{fb}]$ & QCD $[\mathrm{fb}]$ & QCD + EW $_{\text {approx }}[\mathrm{fb}]$ & $\mathrm{QCD} \times \mathrm{EW}_{\text {approx }}[\mathrm{fb}]$ \\
\hline $279.8_{-8.0 \%}^{+7.8 \%}$ & $322.8_{-5.8 \%}^{+7.3 \%}$ & 318.8 & 318.4 \\
\hline
\end{tabular}

Table 4. Fiducial cross sections for pp $\rightarrow \mu^{+} \nu_{\mu} \mathrm{e}^{-} \bar{\nu}_{\mathrm{e}}$, i.e. for the exclusive zero-jet event selection, at $\sqrt{s}=13 \mathrm{TeV}$ for MePs@Lo, MePs@NLo QCD, MePs@Nlo QCD + EW approx and MePs@NLO $\mathrm{QCD} \times \mathrm{EW}_{\text {approx}}$.

\begin{tabular}{|c|c|c|c|}
\hline MePs@Lo & \multicolumn{3}{|c|}{ MePs@NLO } \\
\hline QCD $[\mathrm{fb}]$ & QCD $[\mathrm{fb}]$ & QCD + EW $_{\text {approx }}[\mathrm{fb}]$ & QCD $\times \mathrm{EW}_{\text {approx }}[\mathrm{fb}]$ \\
\hline $108.7_{-10.2 \%}^{+17.6 \%}$ & $131.8_{-6.9 \%}^{+9.6 \%}$ & 129.2 & 129.0 \\
\hline
\end{tabular}

Table 5. Fiducial cross sections for pp $\rightarrow \mu^{+} \nu_{\mu} \mathrm{e}^{-} \bar{\nu}_{\mathrm{e}}$ j, i.e. for the exclusive one-jet event selection, at $\sqrt{s}=13 \mathrm{TeV}$ for MePs@Lo, MePs@NLO QCD, MePs@Nlo QCD + EW approx and MePs@NLO $\mathrm{QCD} \times \mathrm{EW}_{\text {approx}}$.

reader that an overlap of the QED corrections provided by the soft-photon resummation and the approximate EW corrections exists. It does, however, not impact the accuracy of the method in the targeted EW Sudakov regime, cf. the discussion at the end of section 2.4.

As an additional reference, we furthermore compile predictions from merging the LO matrix elements for pp $\rightarrow \mu^{+} \nu_{\mu} \mathrm{e}^{-} \bar{\nu}_{\mathrm{e}}+0,1,2,3 \mathrm{j}$ using the MEPs@Lo approach [35]. As for the MePs@NLO calculations we use $Q_{\text {cut }}=30 \mathrm{GeV}$ here.

In tables 4 and 5 we compile the fiducial cross sections for the various theoretical predictions in the zero- and one-jet selection, respectively. These can be directly compared to the respective fixed-order results quoted in tables 1 and 2. We recognise that the MEPs@Lo cross sections for both event selections are significantly lower (12\% for WW and $33 \%$ for $\mathrm{WWj}$ production) than at fixed order, originating from the inclusion of parton emissions off the respective Born configuration that can trigger the applied jet veto and thus reduce the naïve LO cross section. Both MEPs@NLO QCD cross sections are in very good agreement with the respective fixed-order result (within $2 \%$ for both WW and WWj production). The MePs NLO QCD corrections amount to $+15 \%$ for $\mathrm{WW}$ and $+21 \%$ for WWj. While the scale uncertainty is only marginally reduced by going to NLO QCD for $\mathrm{WW}$, it decreases by almost a factor of two for $\mathrm{WWj}$. The rates for the additive and multiplicative inclusion of approximate EW NLO corrections come out somewhat larger than at fixed order. In fact, for both selections these corrections stay below $-1.5 \%$. Again, it is to note that these EW corrections are tailored to the EW Sudakov regime and are not expected to fully reproduce the exact NLO EW corrections for inclusive observables.

In figures 14 and 15 we display differential distributions for the zero-jet event selection, while results for the one-jet selection are presented in figures 16 and 17 . Finally, in figures 18 and 19 we present predictions for the ratio of differential distributions between $\mathrm{pp} \rightarrow \mu^{+} \nu_{\mu} \mathrm{e}^{-} \bar{\nu}_{\mathrm{e}}$ and $\mathrm{pp} \rightarrow \mu^{+} \nu_{\mu} \mathrm{e}^{-} \bar{\nu}_{\mathrm{e}}$, that have been studied at LO QCD, NLO QCD, $\mathrm{NLO}$ QCD $+\mathrm{EW}$, and NLO QCD $\times$ EW in section 3.1.3. 

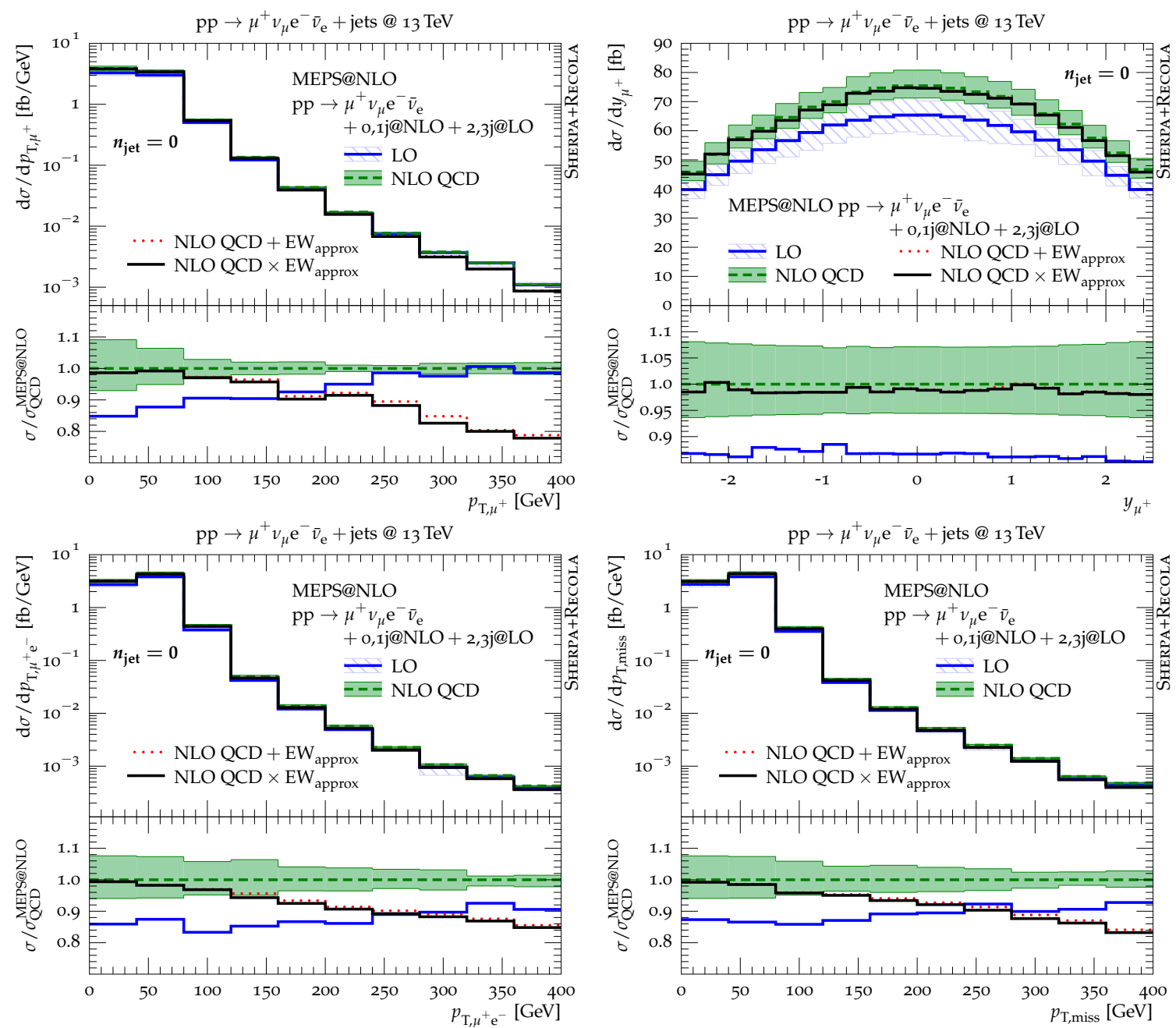

Figure 14. Predictions from multi-jet merged parton-shower simulations for the $n_{\text {jet }}=0$ event selection: transverse momentum of the anti-muon (top left), rapidity of the anti-muon (top right), transverse momentum of the anti-muon-electron system (bottom left), and missing transverse momentum (bottom right). All results contain YFS soft-photon resummation. For the MEPs@NLO calculation we present results including approximate NLO EW corrections in the additive and multiplicative approach.

In all these plots in the upper panels we display the MEPs@NLO predictions, now including YFS soft-photon resummation, as green dashed line including its 7-point scale variation uncertainty band. The corresponding MEPs@Lo predictions are indicated by the blue solid line and the hatched uncertainty band. Furthermore, MEPs@NLO predictions including the EW virtual approximation in its additive (dotted red) and multiplicative (solid black) manner, are provided. In the lower panels we compile the ratios with respect to the MEPs@NLO QCD prediction.

We begin the discussion with the results for the zero-jet event selection. In figures 14 and 15 we recognise, that the inclusion of the NLO QCD matrix elements for the zeroand one-jet processes increases the fiducial cross section by about $15 \%$ as already seen in 

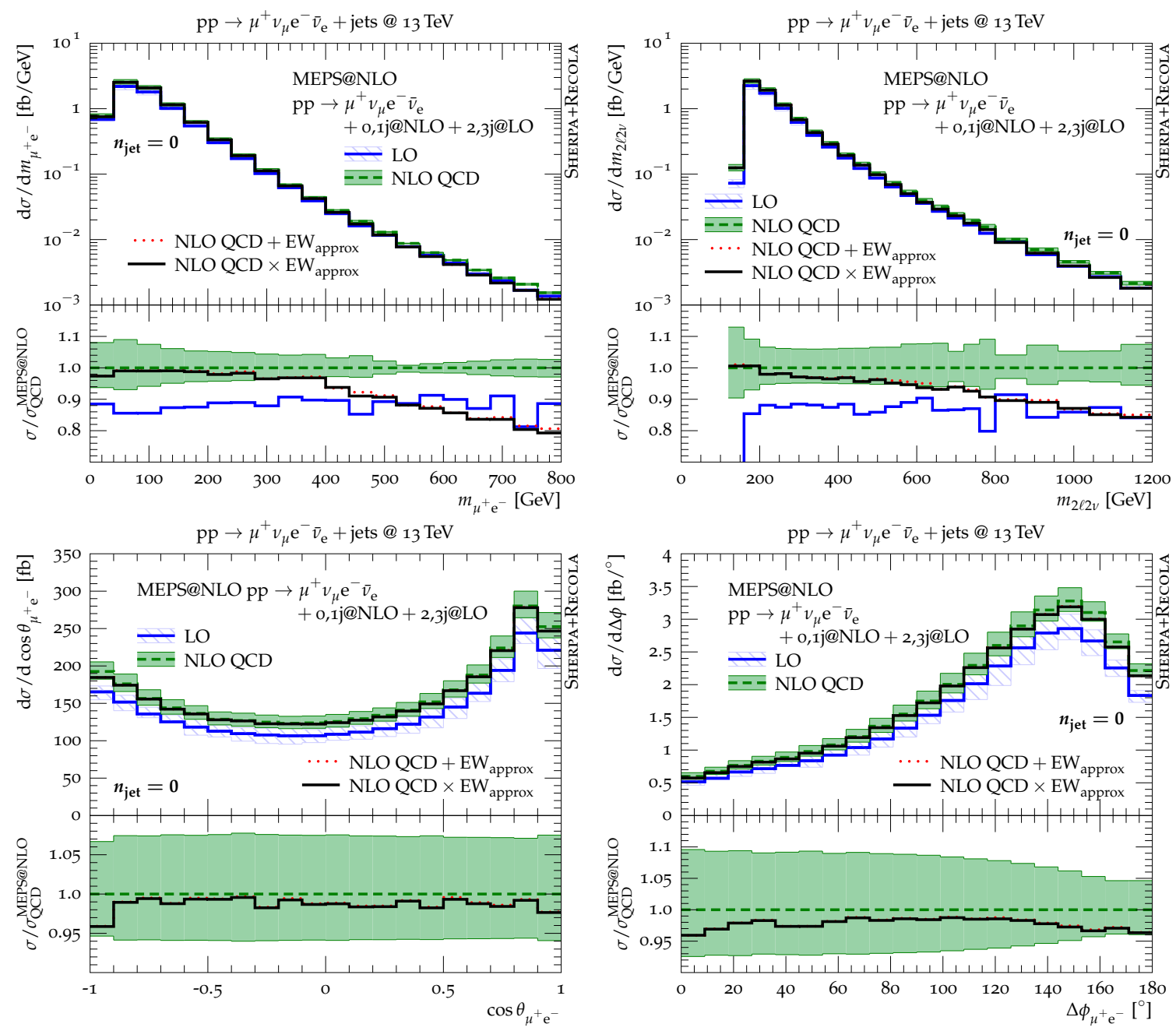

Figure 15. Predictions from multi-jet merged parton-shower simulations for the $n_{\text {jet }}=0$ event selection: invariant mass of the anti-muon and electron (top left), invariant mass of the four leptons (top right), cosine of the angle between the anti-muon and the electron (bottom left), and azimuthalangle distance between the anti-muon and the electron (bottom right). All results contain YFS softphoton resummation. For the MEPs@NLO calculation we present results including approximate NLO EW corrections in the additive and multiplicative approach.

table 4 but has a comparably mild impact on the shapes of differential distributions. Most notably, for the transverse momentum of the anti-muon the shape distortion reaches about $15 \%$, and the QCD corrections decrease with increasing transverse momentum.

Concerning the impact of the approximate NLO EW corrections, two patterns emerge. For the transverse-momentum-type observables, as well as the invariant masses $m_{\mu^{+} \mathrm{e}^{-}}$and $m_{2 \ell 2 \nu}$, EW corrections suppress the high- $p_{\mathrm{T}}$ and high-mass tails, up to about $-20 \%$ for the considered observable ranges as a consequence of enhanced EW logarithmic corrections. For the anti-muon rapidity distributions, as well as the two considered angular observables, i.e. $\cos \theta_{\mu^{+} \mathrm{e}^{-}}$and $\Delta \phi_{\mu^{+} \mathrm{e}^{-}}$, the EW corrections are very small and essentially flat, consistent with the observation for the fixed-order calculations. 


\begin{tabular}{|c|c|c|c|}
\hline MEPs@Lo & \multicolumn{3}{|c|}{ MEPs@NLO } \\
\hline QCD & QCD & QCD $+\mathrm{EW}_{\text {approx }}$ & QCD $\times \mathrm{EW}_{\text {approx }}$ \\
\hline $0.388_{-13.9 \%}^{+20.6 \%}$ & $0.408_{-3.2 \%}^{+4.4 \%}$ & 0.405 & 0.405 \\
\hline
\end{tabular}

Table 6. Ratios of fiducial cross sections between pp $\rightarrow \mu^{+} \nu_{\mu} \mathrm{e}^{-} \bar{\nu}_{\mathrm{e}} \mathrm{j}$ and $\mathrm{pp} \rightarrow \mu^{+} \nu_{\mu} \mathrm{e}^{-} \bar{\nu}_{\mathrm{e}}$ at $\sqrt{s}=13 \mathrm{TeV}$ at MePs@Lo, MePs@Nlo QCD, MePs@Nlo QCD + EW approx and MePs@NLO $\mathrm{QCD} \times \mathrm{EW}_{\text {approx}}$.

However, in contrast to the fixed-order results presented in section 3.1.1, the additive and multiplicative approach of combining QCD and EW corrections here yield very similar results, with the size of the corrections being close to the multiplicative fixed-order scheme. The small difference between the three results is due to several reasons. First of all, the QCD corrections are much smaller in the merged calculation since more contributions are incorporated in the corresponding LO results and on top of that even tend to decrease with increasing transverse momenta where EW corrections are sizeable. Second, the additive combination as defined in eq. (2.11) takes $\mathrm{QCD} \times \mathrm{EW}$ corrections into account via the explicit higher-multiplicity processes and the parton showers.

For the observables in the one-jet event selection, presented in figures 16 and 17, similar conclusions apply. The inclusion of the NLO QCD corrections in the MEPs@NLO calculations increases the fiducial cross section by about $21 \%$ with respect to MePs@Lo, cf. table 5. At the same time the systematic uncertainties get reduced by almost a factor two. In particular, for the transverse-momentum and invariant-mass distributions the NLO QCD corrections have significant impact on the distributions' shape, however, much smaller than for the fixed-order evaluation of the observables. This smaller impact is caused by the inclusion of additional real-radiation processes through the parton shower and the highermultiplicity matrix elements, modelling in particular the jet-veto process more reliably. In fact, for the jet transverse-momentum distribution, the shape is only very mildly affected by the NLO QCD corrections.

For the jet and anti-muon rapidity distribution, as well as the two angular observables, approximative EW corrections are of 1-2\% size only, well within the MEPs@NLO uncertainty bands, and essentially flat. For the $p_{\mathrm{T}}$-type and the invariant-mass distributions sizeable EW Sudakov-logarithmic suppression effects are found, compatible with the observations for the fixed-order results in section 3.1.2.

The large deviations seen between the NLO QCD + EW and NLO QCD $\times$ EW predictions at fixed order are not present in the merged calculations. As in the case of WW production, this is due to the fact that the MEPs@Lo calculation incorporates already a sizeable fraction of the QCD corrections, and that the merged NLO QCD + EW predictions include $\mathrm{QCD} \times \mathrm{EW}$ corrections.

\subsubsection{Ratios of WW and WWj}

Given the MEPs@NLO QCD predictions with and without the inclusion of approximate EW NLO corrections for the exclusive zero- and one-jet event selections, we can now pro- 

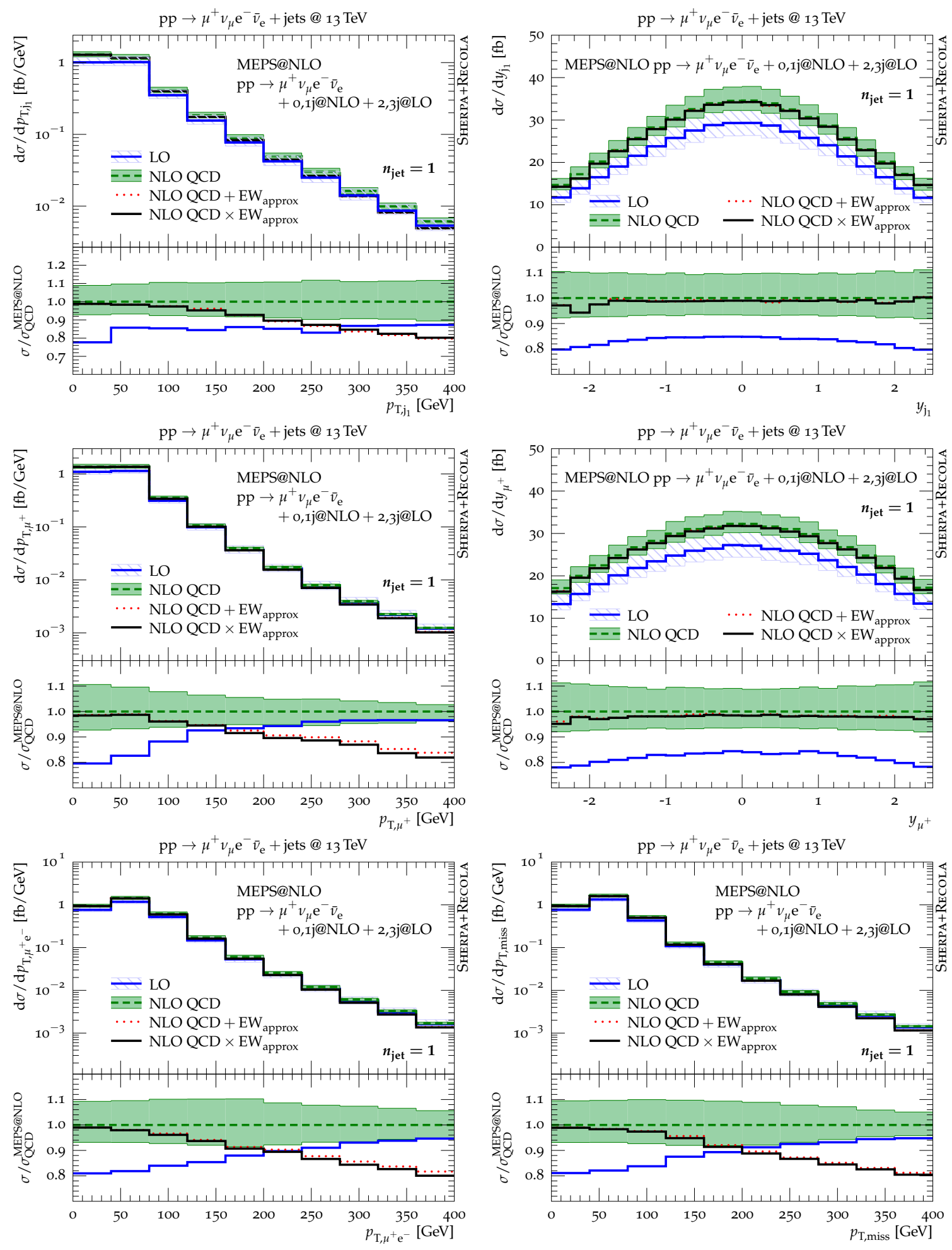

Figure 16. Predictions from multi-jet merged parton-shower simulations for the $n_{\text {jet }}=1$ event selection: transverse momentum of the jet (top left), rapidity of the jet (top right), transverse momentum of the anti-muon (middle left), rapidity of the anti-muon (middle right), transverse momentum of the anti-muon-electron system (bottom left), and missing transverse momentum (bottom right). All results contain YFS soft-photon resummation. For the MEPs@NLO calculation we present results including approximate NLO EW corrections in the additive and multiplicative approach. 

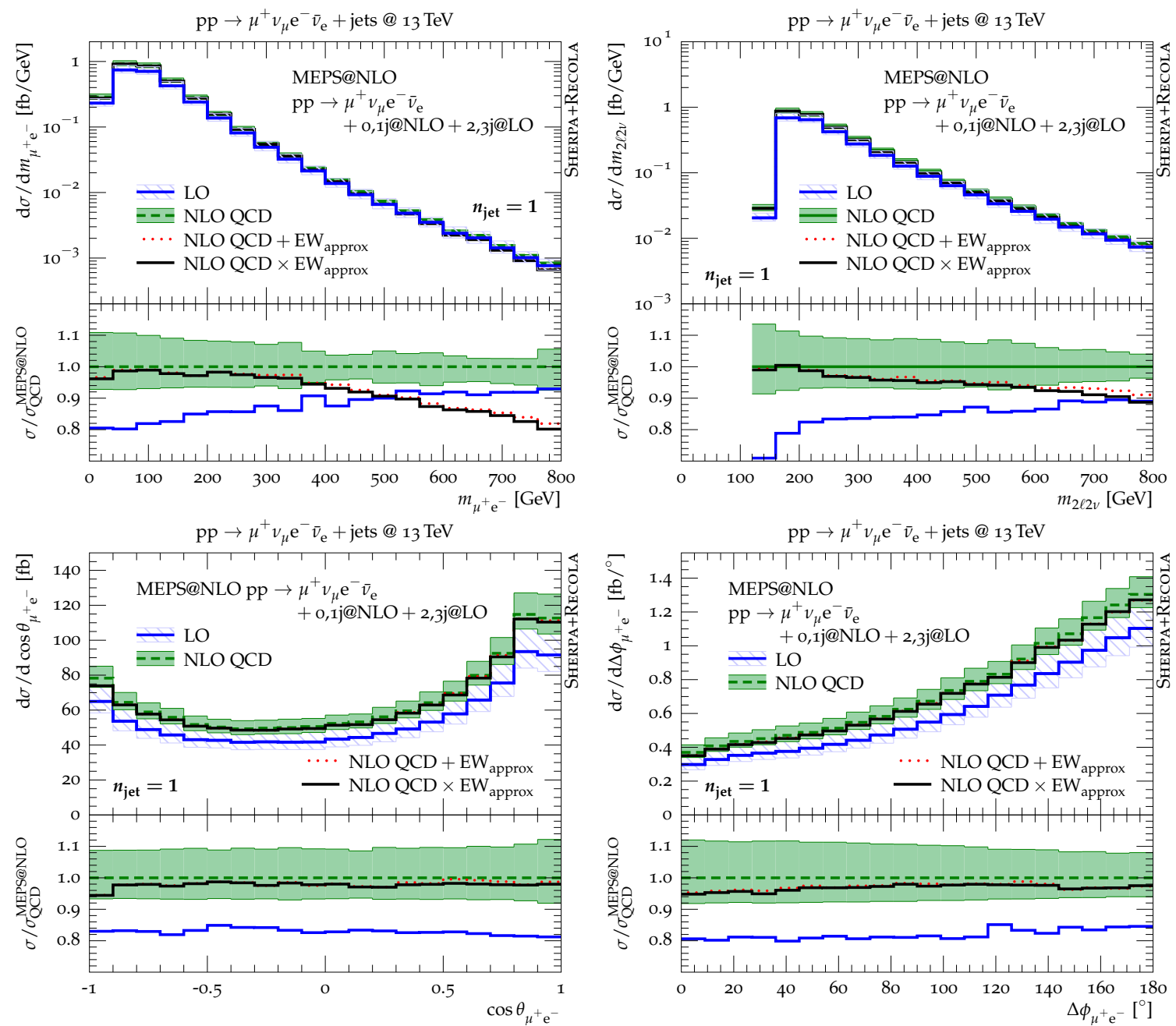

Figure 17. Predictions from multi-jet merged parton-shower simulations for the $n_{\text {jet }}=1$ event selection: invariant mass of the anti-muon and electron (top left), invariant mass of the four leptons (top right), cosine of the angle between the anti-muon and the electron (bottom left), and azimuthalangle distance between the anti-muon and the electron (bottom right). All results contain YFS softphoton resummation. For the MEPs@NLO calculation we present results including approximate NLO EW corrections in the additive and multiplicative approach.

ceed to study ratios of fiducial cross sections and differential distributions. Corresponding fixed-order predictions have been presented in section 3.1.3.

In table 6 we compile the cross-section ratios between pp $\rightarrow \mu^{+} \nu_{\mu} \mathrm{e}^{-} \bar{\nu}_{\mathrm{e}} \mathrm{j}$ and $\mathrm{pp} \rightarrow$ $\mu^{+} \nu_{\mu} \mathrm{e}^{-} \bar{\nu}_{\mathrm{e}}$ for the MePs@Lo, MePs@NLO QCD, MePs@NLO QCD + EW approx , and MEPs@NLO QCD $\times \mathrm{EW}_{\text {approx }}$ calculations. In particular for the MEPs@Lo predictions the ratio is significantly smaller than at LO QCD and closer to the NLO result, cf. table 3. As discussed before, pure LO calculations do not address the applied jet vetoes, while in the MEPs@Lo approach these as well as many higher-order contributions are addressed by the parton shower off the respective Born process and higher-multiplicity matrix elements. In contrast, the MEPs@NLO QCD agrees with its fixed-order equivalent within 1\%. The 

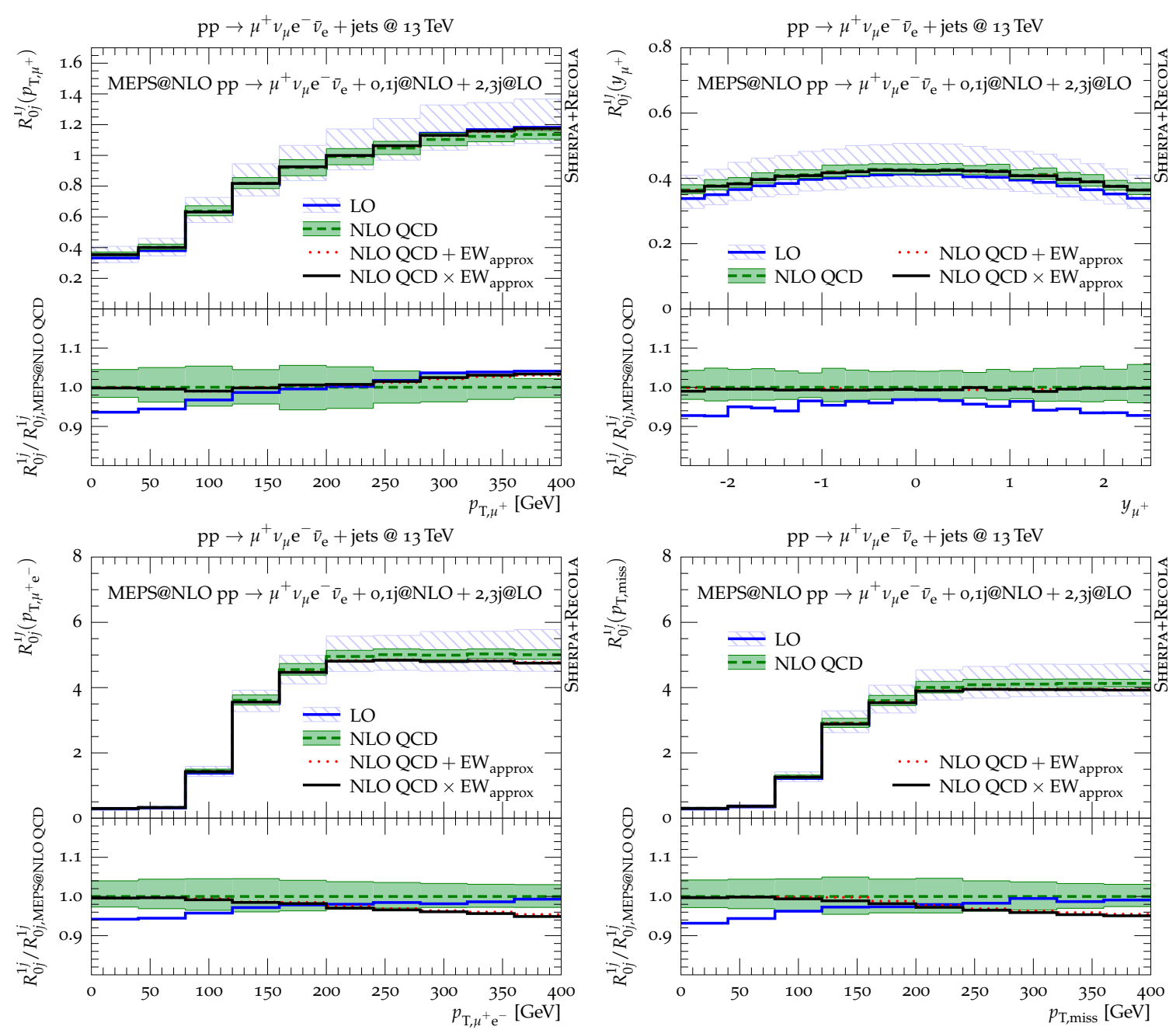

Figure 18. Predictions from multi-jet merged parton-shower simulations for the ratios between differential distributions in the one- and zero-jet event selection: transverse momentum of the anti-muon (top left), rapidity of the anti-muon (top right), transverse momentum of the antimuon-electron system (bottom left), and missing transverse momentum (bottom right). All results contain YFS soft-photon resummation. For the MEPs@NLO calculation we present results including approximate NLO EW corrections in the additive and multiplicative approach.

inclusion of EW corrections in the MEPs@NLO QCD + EW approx and MEPs@NLO QCD $\times$ $\mathrm{EW}_{\text {approx }}$ approach amounts to a reduction of the ratio by less than $1 \%$, respectively, somewhat less than at fixed order.

In figures 18 and 19 we present ratios between differential distributions for the oneand zero-jet selection as defined in eq. (3.5). What we observed for the ratios of fiducial cross sections, is even more prominent in the kinematic distributions. When comparing to figures 8 and 9 we recognise a dramatic difference between the LO QCD and the MEPs@LO prediction. The huge NLO QCD corrections observed before get reduced to at most $10 \%$ for the merged results, with the exception of the low mass region in the $m_{2 \ell 2 \nu}$ distribution. For $m_{2 \ell 2 \nu} \lesssim 2 M_{\mathrm{W}} \approx 161 \mathrm{GeV}$ the production of two resonant $\mathrm{W}$ bosons is not possible, and the 

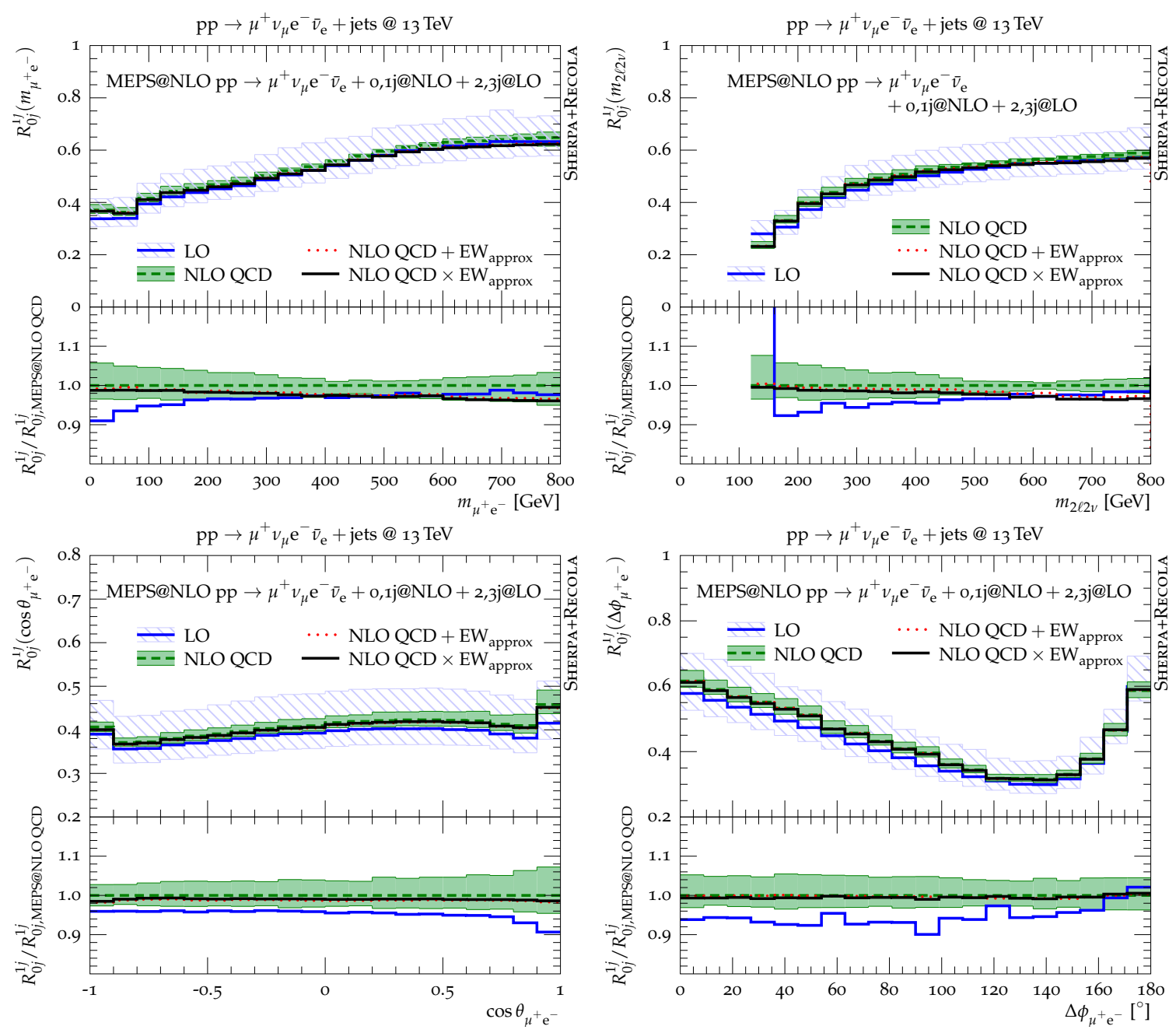

Figure 19. Predictions from multi-jet merged parton-shower simulations for the ratios between differential distributions in the one- and zero-jet event selection: invariant mass of the anti-muon and electron (top left), invariant mass of the four leptons (top right), cosine of the angle between the anti-muon and the electron (bottom left), and azimuthal-angle distance between the antimuon and the electron (bottom right). All results contain YFS soft-photon resummation. For the MEPs@NLO calculation we present results including approximate NLO EW corrections in the additive and multiplicative approach.

effect of singly-resonant diagrams with different kinematics and a huge $K$ factor becomes relevant. In this phase-space region the LO WW cross section is stronger suppressed than the cross sections with real-jet emission. With the exception of this phase-space region, for all the considered distributions the ratios are considerably stabilised by the inclusion of the QCD parton shower and higher-multiplicity matrix elements.

The associated (correlated) scale uncertainties get significantly reduced when going from MEPs@Lo to MEPS@NLO QCD. In particular for the phase-space regions of large transverse momenta and large invariant masses these are much smaller than for the fixedorder evaluations. Apart from the low-mass region in $m_{2 \ell 2 \nu}$ the MEPs@NLO QCD uncertainties remain of order $\pm 5 \%$. 
The approximate NLO EW corrections have only mild impact on the ratios of differential distributions. They largely cancel between numerator and denominator and stay below $5 \%$ also in the tails of the transverse-momentum and invariant-mass distributions. Furthermore, the MEPs@NLO QCD $+\mathrm{EW}_{\text {approx }}$ and MEPs@NLO QCD $\times \mathrm{EW}_{\text {approx }}$ predictions yield almost identical results, in contrast to the NLO QCD + EW fixed-order prediction. This is basically a consequence of the reduced NLO QCD corrections in the merged calculations. Overall, apart from the high- $p_{\mathrm{T}}$ and high-mass tails, the EW corrections stay within the MePs@NLO QCD uncertainty band.

The results for the EW corrections in the merged calculation should be taken with some caution. First, only virtual EW corrections are included exactly, while real EW corrections are integrated out in an approximated way. This approximation is expected to yield good results for observables in the EW Sudakov regime where the kinematic invariants are large with respect to the $\mathrm{W}$-boson mass but not for inclusive observables such as the fiducial cross section. Second, the difference between MEPs@NLO QCD + EW approx and MEPs@NLO QCD $\times \mathrm{EW}_{\text {approx }}$ predictions does not provide a reliable error estimate on the missing $\mathrm{QCD} \times \mathrm{EW}$ corrections since both prescriptions are too close to each other.

\section{Conclusion}

This article provides a combined analysis of $\mathrm{WW}$ and $\mathrm{WWj}$ production at higher order including effects of off-shell and non-resonant contributions, emphasising the combination of QCD and EW corrections. It is the first time that NLO QCD and NLO EW corrections to both pp $\rightarrow \mu^{+} \nu_{\mu} \mathrm{e}^{-} \bar{\nu}_{\mathrm{e}}$ and pp $\rightarrow \mu^{+} \nu_{\mu} \mathrm{e}^{-} \bar{\nu}_{\mathrm{e}} \mathrm{j}$ are presented together in consistent setups. Both processes are analysed while applying jet vetoes in order to avoid large QCD corrections. It is worth noticing that results including NLO EW corrections for the off-shell $\mathrm{WWj}$ production are presented here for the first time. In addition to strictly fixed-order results, merged predictions including different jet multiplicities and parton showers are provided. These are combined in an approximate way with EW corrections in the virtual EW approximation which describes the leading-logarithmic corrections in the Sudakov regime well. All results have been obtained with the SHERPA+RECOLA framework, which is completely automated. Though we did not study their phenomenological impact in this article, non-perturbative corrections to the parton-shower predictions due to hadronisation and multiple-parton interactions can be easily invoked in the SHERPA framework.

After discussing numerical results at fixed order for W-pair production with and without an extra jet separately, ratios of cross sections and differential distributions are presented. For fixed-order calculations, our results (in particular the study of differential crosssection ratios) clearly support a preference for the multiplicative combination of QCD and EW corrections as suggested by the structure of the enhanced EW logarithmic corrections. This further reinforces the use of the approximative EW corrections in merged predictions as it relies on the assumption that processes with different jet multiplicities have similar EW corrections. Nonetheless it should be kept in mind that the virtual approximation catches the dominant corrections in the high-energy limit but does not improve predictions for inclusive observables. In addition to the predictions of the multi-jet merged sample, we 
present comparisons of zero- and one-jet predictions between the fixed-order calculations and the merged ones using two different scale choices. These comparisons emphasise the benefits of the calculation based on multi-jet merging which do not suffer from some of the limitations of the fixed-order calculations. In the end, our study shows that the merged calculations provide more stable predictions, in particular regarding ratios of cross sections and distributions for WWj versus WW production.

Finally, the results presented here are particularly relevant for the experimental measurements at Run 2 and the upcoming high-luminosity phase of the LHC. We hope that these (as well as the corresponding tools) will be fully exploited by both the ATLAS and CMS collaborations.

Note added. The same day the present article appeared on arXiv.org, an independent study on matching NLO corrections to the parton shower in diboson production was made public [94].

\section{Acknowledgments}

We would like to thank Jean-Nicolas Lang for supporting and continuously improving REcolA. SB, SS, and MS received funding from the European Union's Horizon 2020 research and innovation programme as part of the Marie Skłodowska-Curie Innovative Training Network MCnetITN3 (grant agreement no. 722104). AD acknowledges financial support by the German Federal Ministry for Education and Research (BMBF) under contract no. 05H18WWCA1. The research of MP has received funding from the European Research Council (ERC) under the European Union's Horizon 2020 Research and Innovation Programme (grant agreement no. 683211). MS is funded by the Royal Society through a University Research Fellowship. SS acknowledges support through the Fulbright-Cottrell Award and from BMBF (contract no. 05H18MGCA1).

\section{A Fragmentation function}

This appendix is devoted to estimate the numerical impact of a proper IR-safe photon recombination. To that end, fragmentation functions have been implemented in MoCANLO following refs. [29, 31, 32]. The implementation has been validated against the code used in ref. [32] for the computation of EW corrections for $\mathrm{pp} \rightarrow \ell^{+} \ell^{-}$jj. The photon-jet energy fraction

$$
z_{\gamma}=\frac{E_{\gamma}}{E_{\gamma}+E_{a}}
$$

has been taken to be equal to 0.7 , where $E_{\gamma}$ and $E_{a}$ denote the energies of the photon and a QCD parton, respectively. The fit parameters entering the fragmentation function are the ones obtained from ref. [95] and read

$$
\mu_{0}=0.14 \mathrm{GeV} \quad \text { and } \quad C=-13.26 .
$$

In this simulation, the LO only includes QCD partons in the initial state, i.e. contributions with initial-state photons are omitted. Concerning the EW corrections, only photon 


\begin{tabular}{|c|c|c|c|}
\hline$\sigma^{\mathrm{LO}}[\mathrm{fb}]$ & $\sigma_{\mathrm{EW}, \text { cons. }}^{\mathrm{NLO}}[\mathrm{fb}]$ & $\sigma_{\mathrm{EW}, \text { simp. }}^{\mathrm{NLO}}[\mathrm{fb}]$ & $\sigma_{\mathrm{EW}, \text { simp. }}^{\mathrm{NLO}} / \sigma_{\mathrm{EW}, \text { cons. }}^{\mathrm{NLO}}-1[\%]$ \\
\hline $162.545(3)$ & $155.696(5)$ & $155.883(5)$ & 0.12 \\
\hline
\end{tabular}

Table 7. Fiducial cross sections for pp $\rightarrow \mu^{+} \nu_{\mu} \mathrm{e}^{-} \bar{\nu}_{\mathrm{ej}}$ at $\sqrt{s}=13 \mathrm{TeV}$ at LO and NLO EW with (cons.) and without (simp.) proper photon-jet separation. In addition the percentage difference between the latter results is shown.
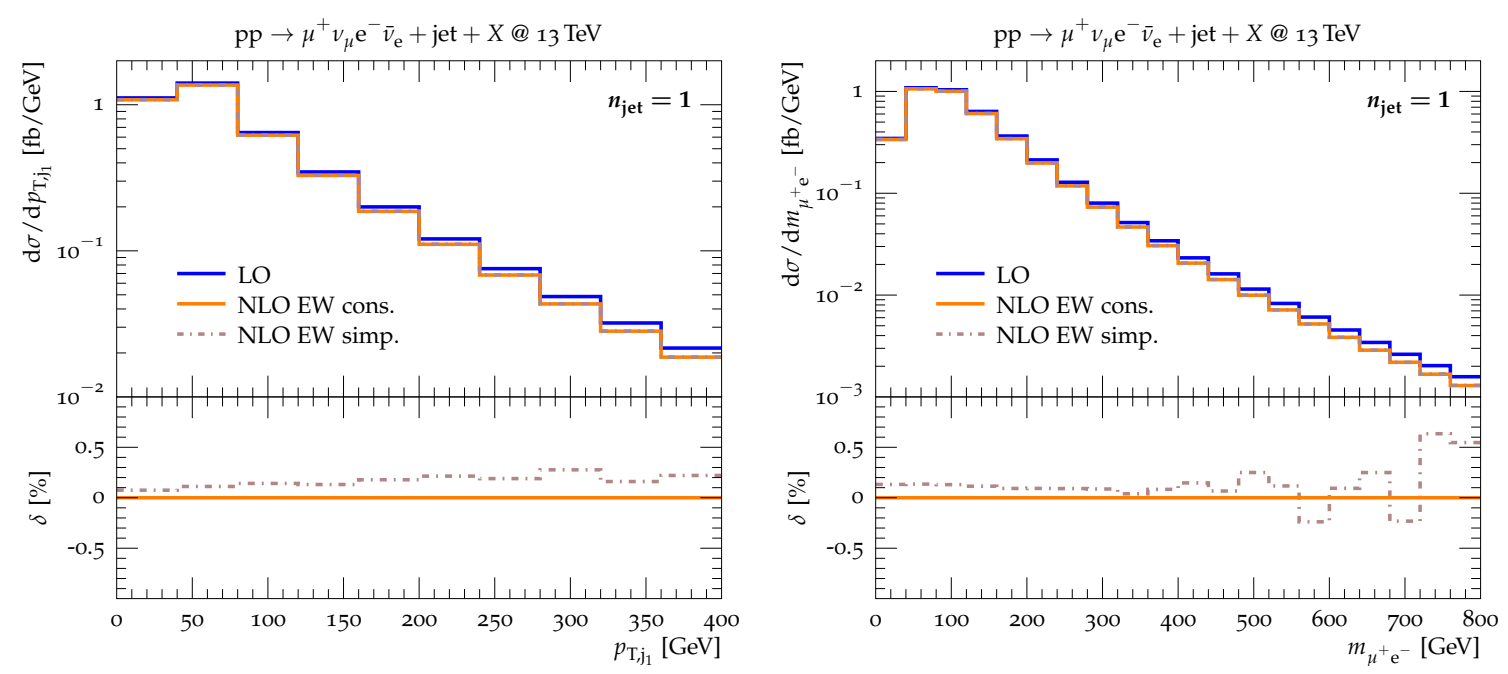

Figure 20. Differential distributions for pp $\rightarrow \mu^{+} \nu_{\mu} \mathrm{e}^{-} \bar{\nu}_{\mathrm{e}} \mathrm{j}$ at $\sqrt{s}=13 \mathrm{TeV}$ at LO and NLO EW: transverse momentum of the hardest jet (left) and invariant mass of the anti-muon-electron system (right). The NLO EW predictions are obtained with (cons.) and without (simp.) proper photon-jet separation. In the lower panels, the relative difference between the NLO EW predictions without and with proper photon-jet separation in per cent is shown.

radiation and EW virtual corrections are considered, while interference contributions in the real radiation from matrix elements at different orders in the couplings are not taken into account.

In table 7, NLO EW cross sections with consistent inclusion of photon-jet separation (cons.) and in the simplified set-up of section 2.6 (simp.) are given. The difference between these two prescriptions is about a per mille only. In addition, in figure 20 two differential distributions are shown in both set-ups: the distribution in the transverse momentum of the hardest jet (left) and the distribution in the invariant mass of the anti-muon-electron system (right).

For large transverse momentum or large invariant mass, the shape of the ratio of the distributions in both methods is dominated by the Monte Carlo statistical error. Disregarding these fluctuations, one notices differences of a few per mille for the transverse-momentum distribution and of only about one per mille for the invariant-mass distribution. This reduced effect can be explained by the fact that leptonic observables are only indirectly sensitive to effects from photon-jet separation. Overall, this analysis indicates that the effect of a consistent treatment of photon-jet separation is rather small for our calculational set-up. This justifies the simplified approach that we have taken. 
Moreover, we investigated the dependence of the simplified approach on the technical cuts used in the MOCANLO generator. We did not observe a dependence beyond the per-mille level for reasonable parameter values.

Open Access. This article is distributed under the terms of the Creative Commons Attribution License (CC-BY 4.0), which permits any use, distribution and reproduction in any medium, provided the original author(s) and source are credited.

\section{References}

[1] ATLAS collaboration, Measurement of fiducial and differential $W^{+} W^{-}$production cross-sections at $\sqrt{s}=13 \mathrm{TeV}$ with the ATLAS detector, Eur. Phys. J. C 79 (2019) 884 [arXiv: 1905.04242] [INSPIRE].

[2] CMS collaboration, Search for anomalous couplings in boosted WW/WZ $\rightarrow \ell \nu \mathrm{q} \overline{\mathrm{q}}$ production in proton-proton collisions at $\sqrt{s}=8$ TeV, Phys. Lett. B 772 (2017) 21 [arXiv:1703.06095] [INSPIRE].

[3] ATLAS collaboration, Measurement of $W^{+} W^{-}$production in association with one jet in proton-proton collisions at $\sqrt{s}=8 \mathrm{TeV}$ with the ATLAS detector, Phys. Lett. B 763 (2016) 114 [arXiv: 1608.03086] [INSPIRE].

[4] J. Ohnemus, Order- $\alpha_{\mathrm{s}}$ calculation of hadronic $W^{-} W^{+}$production, Phys. Rev. D 44 (1991) 1403 [INSPIRE].

[5] U. Baur, T. Han and J. Ohnemus, QCD corrections and nonstandard three vector boson couplings in $W^{+} W^{-}$production at hadron colliders, Phys. Rev. D 53 (1996) 1098 [hep-ph/9507336] [INSPIRE].

[6] J.M. Campbell and R. Ellis, An update on vector boson pair production at hadron colliders, Phys. Rev. D 60 (1999) 113006 [hep-ph/9905386] [INSPIRE].

[7] S. Frixione and B.R. Webber, Matching NLO QCD computations and parton shower simulations, JHEP 06 (2002) 029 [hep-ph/0204244] [INSPIRE].

[8] K. Hamilton, A positive-weight next-to-leading order simulation of weak boson pair production, JHEP 01 (2011) 009 [arXiv:1009.5391] [INSPIRE].

[9] J.H. Kühn, F. Metzler, A.A. Penin and S. Uccirati, Next-to-Next-to-Leading Electroweak Logarithms for W-Pair Production at LHC, JHEP 06 (2011) 143 [arXiv:1101.2563] [INSPIRE].

[10] A. Bierweiler, T. Kasprzik, J.H. Kühn and S. Uccirati, Electroweak corrections to W-boson pair production at the LHC, JHEP 11 (2012) 093 [arXiv:1208.3147] [INSPIRE].

[11] J. Baglio, L.D. Ninh and M.M. Weber, Massive gauge boson pair production at the LHC: a next-to-leading order story, Phys. Rev. D 88 (2013) 113005 [Erratum ibid. 94 (2016) 099902] [arXiv: 1307.4331] [INSPIRE].

[12] S. Gieseke, T. Kasprzik and J.H. Kühn, Vector-boson pair production and electroweak corrections in HERWIG++, Eur. Phys. J. C 74 (2014) 2988 [arXiv:1401.3964] [inSPIRE].

[13] B. Biedermann et al., Next-to-leading-order electroweak corrections to $p p \rightarrow W^{+} W^{-} \rightarrow 4$ leptons at the LHC, JHEP 06 (2016) 065 [arXiv: 1605.03419] [INSPIRE]. 
[14] S. Kallweit, J.M. Lindert, S. Pozzorini and M. Schönherr, NLO QCD+EW predictions for $2 \ell 2 \nu$ diboson signatures at the LHC, JHEP 11 (2017) 120 [arXiv:1705.00598] [INSPIRE].

[15] T. Gehrmann et al., $W^{+} W^{-}$Production at Hadron Colliders in Next to Next to Leading Order QCD, Phys. Rev. Lett. 113 (2014) 212001 [arXiv:1408.5243] [InSPIRE].

[16] M. Grazzini, S. Kallweit, S. Pozzorini, D. Rathlev and M. Wiesemann, $W^{+} W^{-}$production at the LHC: fiducial cross sections and distributions in NNLO QCD, JHEP 08 (2016) 140 [arXiv: 1605.02716] [INSPIRE].

[17] M. Grazzini, S. Kallweit, J.M. Lindert, S. Pozzorini and M. Wiesemann, NNLO QCD + NLO EW with Matrix +OpenLoops: precise predictions for vector-boson pair production, JHEP 02 (2020) 087 [arXiv: 1912.00068] [INSPIRE].

[18] E. Re, M. Wiesemann and G. Zanderighi, NNLOPS accurate predictions for $W^{+} W^{-}$ production, JHEP 12 (2018) 121 [arXiv:1805.09857] [INSPIRE].

[19] M. Grazzini, S. Kallweit, D. Rathlev and M. Wiesemann, Transverse-momentum resummation for vector-boson pair production at NNLL+NNLO, JHEP 08 (2015) 154 [arXiv: 1507.02565] [INSPIRE].

[20] F. Caola, K. Melnikov, R. Röntsch and L. Tancredi, QCD corrections to $W^{+} W^{-}$production through gluon fusion, Phys. Lett. B 754 (2016) 275 [arXiv:1511.08617] [INSPIRE].

[21] M. Grazzini, S. Kallweit, M. Wiesemann and J.Y. Yook, $W^{+} W^{-}$production at the LHC: NLO QCD corrections to the loop-induced gluon fusion channel, Phys. Lett. B 804 (2020) 135399 [arXiv:2002.01877] [INSPIRE].

[22] S. Kallweit, E. Re, L. Rottoli and M. Wiesemann, Accurate single- and double-differential resummation of colour-singlet processes with MATRIX+RadISH: $W^{+} W^{-}$production at the LHC, arXiv:2004.07720 [INSPIRE].

[23] S. Dittmaier, S. Kallweit and P. Uwer, $N L O$ QCD corrections to $p p / p \bar{p} \rightarrow W W+j e t+X$ including leptonic W-boson decays, Nucl. Phys. B 826 (2010) 18 [arXiv:0908.4124] [INSPIRE].

[24] F. Cascioli, S. Höche, F. Krauss, P. Maierhöfer, S. Pozzorini and F. Siegert, Precise Higgs-background predictions: merging NLO QCD and squared quark-loop corrections to four-lepton + 0,1 jet production, JHEP 01 (2014) 046 [arXiv:1309.0500] [INSPIRE].

[25] K. Hamilton, T. Melia, P.F. Monni, E. Re and G. Zanderighi, Merging $W W$ and $W W+j e t$ with MINLO, JHEP 09 (2016) 057 [arXiv: 1606.07062] [INSPIRE].

[26] W.-H. Li, R.-Y. Zhang, W.-G. Ma, L. Guo, X.-Z. Li and Y. Zhang, NLO QCD and electroweak corrections to $W W+$ jet production with leptonic $W$-boson decays at LHC, Phys. Rev. D 92 (2015) 033005 [arXiv: 1507.07332] [INSPIRE].

[27] S. Kallweit, J.M. Lindert, P. Maierhöfer, S. Pozzorini and M. Schönherr, $N L O Q C D+E W$ predictions for $V+$ jets including off-shell vector-boson decays and multijet merging, JHEP 04 (2016) 021 [arXiv: 1511.08692] [INSPIRE].

[28] B. Biedermann, S. Bräuer, A. Denner, M. Pellen, S. Schumann and J.M. Thompson, Automation of NLO QCD and EW corrections with Sherpa and Recola, Eur. Phys. J. C 77 (2017) 492 [arXiv: 1704.05783] [INSPIRE].

[29] A. Denner, S. Dittmaier, T. Kasprzik and A. Mück, Electroweak corrections to $W+$ jet hadroproduction including leptonic W-boson decays, JHEP 08 (2009) 075 [arXiv:0906.1656] [INSPIRE]. 
[30] A. Denner, S. Dittmaier, T. Gehrmann and C. Kurz, Electroweak corrections to hadronic event shapes and jet production in $e^{+} e^{-}$annihilation, Nucl. Phys. B 836 (2010) 37 [arXiv: 1003.0986] [INSPIRE].

[31] A. Denner, S. Dittmaier, T. Kasprzik and A. Mück, Electroweak corrections to dilepton + jet production at hadron colliders, JHEP 06 (2011) 069 [arXiv:1103.0914] [INSPIRE].

[32] A. Denner, L. Hofer, A. Scharf and S. Uccirati, Electroweak corrections to lepton pair production in association with two hard jets at the LHC, JHEP 01 (2015) 094 [arXiv: 1411.0916] [INSPIRE].

[33] A. Denner, S. Dittmaier, P. Maierhöfer, M. Pellen and C. Schwan, QCD and electroweak corrections to WZ scattering at the LHC, JHEP 06 (2019) 067 [arXiv:1904.00882] [INSPIRE].

[34] A. Denner, S. Dittmaier, M. Pellen and C. Schwan, Low-virtuality photon transitions $\gamma^{*} \rightarrow f \bar{f}$ and the photon-to-jet conversion function, Phys. Lett. B 798 (2019) 134951 [arXiv: 1907.02366] [INSPIRE].

[35] S. Höche, F. Krauss, S. Schumann and F. Siegert, QCD matrix elements and truncated showers, JHEP 05 (2009) 053 [arXiv:0903.1219] [INSPIRE].

[36] S. Höche, F. Krauss, M. Schönherr and F. Siegert, NLO matrix elements and truncated showers, JHEP 08 (2011) 123 [arXiv:1009.1127] [INSPIRE].

[37] S. Höche, F. Krauss, M. Schönherr and F. Siegert, QCD matrix elements + parton showers: The NLO case, JHEP 04 (2013) 027 [arXiv:1207.5030] [INSPIRE].

[38] T. Gehrmann, S. Höche, F. Krauss, M. Schönherr and F. Siegert, NLO QCD matrix elements + parton showers in $e^{+} e^{-} \rightarrow$ hadrons, JHEP 01 (2013) 144 [arXiv:1207.5031] [INSPIRE].

[39] S. Höche, F. Krauss, S. Pozzorini, M. Schönherr, J.M. Thompson and K.C. Zapp, Triple vector boson production through Higgs-Strahlung with NLO multijet merging, Phys. Rev. D 89 (2014) 093015 [arXiv: 1403.7516] [INSPIRE].

[40] S. Catani, F. Krauss, R. Kuhn and B.R. Webber, QCD matrix elements + parton showers, JHEP 11 (2001) 063 [hep-ph/0109231] [INSPIRE].

[41] S. Höche, F. Krauss, M. Schönherr and F. Siegert, A critical appraisal of NLO+PS matching methods, JHEP 09 (2012) 049 [arXiv:1111.1220] [INSPIRE].

[42] S. Höche, F. Krauss, M. Schönherr and F. Siegert, $W+n$-jet predictions at the Large Hadron Collider at next-to-leading order matched with a parton shower, Phys. Rev. Lett. 110 (2013) 052001 [arXiv: 1201.5882] [INSPIRE].

[43] S. Höche and M. Schönherr, Uncertainties in next-to-leading order plus parton shower matched simulations of inclusive jet and dijet production, Phys. Rev. D 86 (2012) 094042 [arXiv: 1208.2815] [INSPIRE].

[44] K. Danziger, Efficiency Improvements in Monte Carlo Algorithms for High-Multiplicity Processes. Ph.D. thesis, presented 31 March 2020, .https://cds.cern.ch/record/2715727.

[45] C. Gütschow, J.M. Lindert and M. Schönherr, Multi-jet merged top-pair production including electroweak corrections, Eur. Phys. J. C 78 (2018) 317 [arXiv:1803.00950] [INSPIRE].

[46] M. Schönherr, An automated subtraction of NLO EW infrared divergences, Eur. Phys. J. C 78 (2018) 119 [arXiv:1712.07975] [INSPIRE]. 
[47] M. Schönherr, Next-to-leading order electroweak corrections to off-shell $W W W$ production at the LHC, JHEP 07 (2018) 076 [arXiv: 1806. 00307] [INSPIRE].

[48] M. Reyer, M. Schönherr and S. Schumann, Full NLO corrections to 3-jet production and $\mathbf{R}_{32}$ at the LHC, Eur. Phys. J. C 79 (2019) 321 [arXiv: 1902.01763] [InSPIRE].

[49] N. Baberuxki, C.T. Preuss, D. Reichelt and S. Schumann, Resummed predictions for jet-resolution scales in multijet production in $e^{+} e^{-}$annihilation, JHEP 04 (2020) 112 [arXiv: 1912.09396] [INSPIRE].

[50] SherPa collaboration, Event Generation with Sherpa 2.2, SciPost Phys. 7 (2019) 034 [arXiv: 1905.09127] [INSPIRE].

[51] T. Gleisberg et al., Event generation with SHERPA 1.1, JHEP 02 (2009) 007 [arXiv: 0811.4622] [INSPIRE].

[52] T. Gleisberg and S. Höche, Comix, a new matrix element generator, JHEP 12 (2008) 039 [arXiv: 0808.3674] [INSPIRE].

[53] F. Krauss, R. Kuhn and G. Soff, AMEGIC++ 1.0: A matrix element generator in $\mathrm{C}++$, JHEP 02 (2002) 044 [hep-ph/0109036] [INSPIRE].

[54] T. Gleisberg and F. Krauss, Automating dipole subtraction for QCD NLO calculations, Eur. Phys. J. C 53 (2008) 501 [arXiv:0709.2881] [InSPIRE].

[55] S. Schumann and F. Krauss, A parton shower algorithm based on Catani-Seymour dipole factorisation, JHEP 03 (2008) 038 [arXiv:0709.1027] [INSPIRE].

[56] D.R. Yennie, S.C. Frautschi and H. Suura, The infrared divergence phenomena and high-energy processes, Annals Phys. 13 (1961) 379 [INSPIRE].

[57] M. Schönherr and F. Krauss, Soft Photon Radiation in Particle Decays in SHERPA, JHEP 12 (2008) 018 [arXiv: 0810.5071] [INSPIRE].

[58] S. Actis, A. Denner, L. Hofer, J.-N. Lang, A. Scharf and S. Uccirati, RECOLA: REcursive Computation of One-Loop Amplitudes, Comput. Phys. Commun. 214 (2017) 140 [arXiv: 1605.01090] [INSPIRE].

[59] S. Actis, A. Denner, L. Hofer, A. Scharf and S. Uccirati, Recursive generation of one-loop amplitudes in the Standard Model, JHEP 04 (2013) 037 [arXiv: 1211.6316] [INSPIRE].

[60] A. Denner, S. Dittmaier and L. Hofer, COLLIER - A fortran-library for one-loop integrals, PoS LL2014 (2014) 071 [arXiv: 1407.0087] [InSPIRE].

[61] A. Denner, S. Dittmaier and L. Hofer, COLLIER: a fortran-based Complex One-Loop LIbrary in Extended Regularizations, Comput. Phys. Commun. 212 (2017) 220 [arXiv: 1604.06792] [INSPIRE].

[62] G. 't Hooft and M.J.G. Veltman, Scalar One Loop Integrals, Nucl. Phys. B 153 (1979) 365 [INSPIRE].

[63] W. Beenakker and A. Denner, Infrared Divergent Scalar Box Integrals with Applications in the Electroweak Standard Model, Nucl. Phys. B 338 (1990) 349 [inSPIRE].

[64] S. Dittmaier, Separation of soft and collinear singularities from one loop $N$ point integrals, Nucl. Phys. B 675 (2003) 447 [hep-ph/0308246] [INSPIRE].

[65] A. Denner and S. Dittmaier, Scalar one-loop 4-point integrals, Nucl. Phys. B 844 (2011) 199 [arXiv: 1005.2076] [INSPIRE]. 
[66] G. Passarino and M.J.G. Veltman, One Loop Corrections for $e^{+} e^{-}$Annihilation Into $\mu^{+} \mu^{-}$ in the Weinberg Model, Nucl. Phys. B 160 (1979) 151 [InSPIRE].

[67] A. Denner and S. Dittmaier, Reduction of one loop tensor five point integrals, Nucl. Phys. B 658 (2003) 175 [hep-ph/0212259] [INSPIRE].

[68] A. Denner and S. Dittmaier, Reduction schemes for one-loop tensor integrals, Nucl. Phys. B 734 (2006) 62 [hep-ph/0509141] [INSPIRE].

[69] A. Denner, J.-N. Lang and S. Uccirati, RECOLA2: REcursive Computation of One-Loop Amplitudes 2, Comput. Phys. Commun. 224 (2018) 346 [arXiv:1711.07388] [InSPIRE].

[70] M. Chiesa, A. Denner, J.-N. Lang and M. Pellen, An event generator for same-sign W-boson scattering at the LHC including electroweak corrections, Eur. Phys. J. C 79 (2019) 788 [arXiv: 1906.01863] [INSPIRE].

[71] B. Biedermann, A. Denner and M. Pellen, Large electroweak corrections to vector-boson scattering at the Large Hadron Collider, Phys. Rev. Lett. 118 (2017) 261801 [arXiv: 1611.02951] [INSPIRE].

[72] B. Biedermann, A. Denner and M. Pellen, Complete NLO corrections to $W^{+} W^{+}$scattering and its irreducible background at the LHC, JHEP 10 (2017) 124 [arXiv:1708.00268] [INSPIRE].

[73] S. Kallweit, J.M. Lindert, P. Maierhöfer, S. Pozzorini and M. Schönherr, NLO electroweak automation and precise predictions for W+multijet production at the LHC, JHEP 04 (2015) 012 [arXiv: 1412.5157] [INSPIRE].

[74] M. Chiesa, N. Greiner, M. Schönherr and F. Tramontano, Electroweak corrections to diphoton plus jets, JHEP 10 (2017) 181 [arXiv:1706.09022] [INSPIRE].

[75] N. Greiner and M. Schönherr, NLO QCD $E W$ corrections to diphoton production in association with a vector boson, JHEP 01 (2018) 079 [arXiv:1710.11514] [INSPIRE].

[76] J. Andersen et al., Les Houches 201\%: Physics at TeV Colliders Standard Model Working Group Report, in proceedings of 2017 Les Houches Workshop on Physics at TeV Colliders, Les Houches, France, 5-23 June 2017, arXiv:1803.07977.

[77] NNPDF collaboration, Illuminating the photon content of the proton within a global PDF analysis, SciPost Phys. 5 (2018) 008 [arXiv:1712.07053] [InSPIRE].

[78] A. Buckley et al., LHAPDF6: parton density access in the LHC precision era, Eur. Phys. J. $C \mathbf{7 5}$ (2015) 132 [arXiv:1412.7420] [INSPIRE].

[79] A. Manohar, P. Nason, G.P. Salam and G. Zanderighi, How bright is the proton? A precise determination of the photon parton distribution function, Phys. Rev. Lett. 117 (2016) 242002 [arXiv: 1607.04266] [INSPIRE].

[80] A. Denner, S. Dittmaier, M. Roth and D. Wackeroth, Electroweak radiative corrections to $e^{+} e^{-} \rightarrow W W \rightarrow 4$ fermions in double pole approximation: The RACOONWW approach, Nucl. Phys. B 587 (2000) 67 [hep-ph/0006307] [INSPIRE].

[81] S. Dittmaier and M. Krämer, Electroweak radiative corrections to $W$ boson production at hadron colliders, Phys. Rev. D 65 (2002) 073007 [hep-ph/0109062] [INSPIRE].

[82] LHC Higgs Cross Section Working Group collaboration, Handbook of LHC Higgs Cross Sections: 3. Higgs Properties, arXiv:1307.1347 [INSPIRE]. 
[83] D. Bardin, A. Leike, T. Riemann and M. Sachwitz, Energy Dependent Width Effects in $e^{+} e^{-}$ Annihilation Near the Z Boson Pole, Phys. Lett. B 206 (1988) 539 [inSPIRE].

[84] M. Cacciari, G.P. Salam and G. Soyez, The anti- $k_{t}$ jet clustering algorithm, JHEP 04 (2008) 063 [arXiv:0802.1189] [INSPIRE].

[85] A. Buckley et al., Rivet user manual, Comput. Phys. Commun. 184 (2013) 2803 [arXiv: 1003.0694] [INSPIRE].

[86] E. Bothmann, M. Schönherr and S. Schumann, Reweighting QCD matrix-element and parton-shower calculations, Eur. Phys. J. C 76 (2016) 590 [arXiv: 1606. 08753] [INSPIRE].

[87] A. Denner and S. Pozzorini, One loop leading logarithms in electroweak radiative corrections. 1. Results, Eur. Phys. J. C 18 (2001) 461 [hep-ph/0010201] [INSPIRE].

[88] J.-y. Chiu, R. Kelley and A.V. Manohar, Electroweak Corrections using Effective Field Theory: Applications to the LHC, Phys. Rev. D 78 (2008) 073006 [arXiv:0806.1240] [INSPIRE].

[89] J.-y. Chiu, A. Fuhrer, R. Kelley and A.V. Manohar, Soft and Collinear Functions for the Standard Model, Phys. Rev. D 81 (2010) 014023 [arXiv: 0909.0947] [INSPIRE].

[90] J.R. Andersen et al., Les Houches 2015: Physics at TeV Colliders Standard Model Working Group Report, in 9th Les Houches Workshop on Physics at TeV Colliders, Les Houches, France, 1-19 June 2015, arXiv: 1605.04692 [INSPIRE].

[91] S. Höche, J. Huang, G. Luisoni, M. Schönherr and J. Winter, Zero and one jet combined next-to-leading order analysis of the top quark forward-backward asymmetry, Phys. Rev. D 88 (2013) 014040 [arXiv: 1306.2703] [INSPIRE].

[92] S. Höche, F. Krauss and M. Schönherr, Uncertainties in MEPS@NLO calculations of $h+j e t s$, Phys. Rev. D 90 (2014) 014012 [arXiv:1401.7971] [InSPIRE].

[93] S. Höche, F. Krauss, P. Maierhöfer, S. Pozzorini, M. Schönherr and F. Siegert, Next-to-leading order QCD predictions for top-quark pair production with up to two jets merged with a parton shower, Phys. Lett. B 748 (2015) 74 [arXiv:1402.6293] [INSPIRE].

[94] M. Chiesa, C. Oleari and E. Re, NLO QCD+NLO EW corrections to diboson production matched to parton shower, Eur. Phys. J. C 80 (2020) 849 [arXiv:2005.12146] [INSPIRE].

[95] ALEPH collaboration, First measurement of the quark to photon fragmentation function, $Z$. Phys. C 69 (1996) 365 [INSPIRE]. 